\title{
Exponential separation and principal Floquet bundles for linear parabolic equations on $\mathbb{R}^{N}$
}

\author{
Juraj Húska, Peter Poláčik ${ }^{\dagger}$ \\ School of Mathematics, University of Minnesota \\ Minneapolis, MN 55455
}

\begin{abstract}
We consider linear nonautonomous second order parabolic equations on $\mathbb{R}^{N}$. Under an instability condition, we prove the existence of two complementary Floquet bundles, one spanned by a positive entire solution - the principal Floquet bundle, the other one consisting of sign-changing solutions. We establish an exponential separation between the two bundles, showing in particular that a class of signchanging solutions are exponentially dominated by positive solutions.
\end{abstract}

Keywords: Parabolic equations on $\mathbb{R}^{N}$, exponential separation, positive entire solutions, principal Floquet bundle.

\section{Contents}

1 Introduction 2

2 Main results $\quad 4$

2.1 Standing hypotheses and some terminology . . . . . . . . . . . 4

2.2 Statement of the main results . . . . . . . . . . . . . 6

3 Preliminary results

*Supported in part by Doctoral Dissertation Fellowship of the University of Minnesota

${ }^{\dagger}$ Supported in part by NSF Grant DMS-0400702 
5 Proof of Theorem $2.2 \quad 17$

6 Proof of Theorem $2.4 \quad 29$

7 Proofs of Proposition 2.5 and Theorem $2.6 \quad 37$

8 A remark on the hypotheses $\quad 39$

9 Generalizations $\quad 41$

\section{Introduction}

In this paper we consider the linear nonautonomous parabolic equation

$$
u_{t}=\Delta u+a(x, t) u \quad \text { in } \quad \mathbb{R}^{N} \times J,
$$

where $a \in L^{\infty}\left(\mathbb{R}^{N} \times \mathbb{R}\right)$ and $J=(s, \infty)$ for some $s \in \mathbb{R}$ or $J=\mathbb{R}$. We have chosen (1.1) as a model for a class of more general equations of the form

$$
u_{t}=L(x, t) u \quad \text { in } \quad \mathbb{R}^{N} \times J
$$

where $L(x, t)$ is a time-dependent second-order elliptic operator of divergence form with sufficiently regular coefficients. While the simple form of (1.1) allows us to explain our results and methods without a notational burden, it is representative enough for the whole class (1.2). Indeed, as we demonstrate in the last section, our methods are straightforward to adapt to the more general setting.

If $\mathbb{R}^{N}$ in (1.1) is replaced by $\Omega$, a bounded domain in $\mathbb{R}^{N}$, and (1.1) is complemented with a suitable boundary condition, there is a number of papers devoted to properties of solutions of (1.1) analogous to properties of principal eigenfunctions of time-independent (elliptic) or time-periodic parabolic problems, see for example $[11,12,14,15,16,24,25,26,29,32,33]$. Typical results can briefly be summarized as follows. Let $X$ be a suitable state space for (1.1) (a Banach space in which the initial-boundary value problem is well posed). Then $X$ can be written as the direct sum

$$
X=X^{1}(t) \oplus X^{2}(t) \quad(t \in \mathbb{R}),
$$


where the time-dependent subspaces $X^{1}(t), X^{2}(t), t \in \mathbb{R}$, have the following properties. The space $X^{1}(t)$ is the (one-dimensional) span of a positive function, $X^{2}(t) \backslash\{0\}$ does not contain any nonnegative function; the families $X^{i}(t), t \in \mathbb{R}, i=1,2$, are invariant for $(1.1)$ : if $u_{1}(\cdot, t), u_{2}(\cdot, t)$ are solutions with $u_{i}(\cdot, s) \in X^{i}(s)$, then $u_{i}(\cdot, t) \in X^{i}(t)$ for all $t>s$. Finally, for any such solutions, assuming $u_{1}$ is nontrivial, we have the exponential separation estimate

$$
\frac{\left\|u_{2}(\cdot, t)\right\|_{X}}{\left\|u_{1}(\cdot, t)\right\|_{X}} \leq C e^{-\gamma(t-s)} \frac{\left\|u_{2}(\cdot, s)\right\|_{X}}{\left\|u_{1}(\cdot, s)\right\|_{X}} \quad(t \geq s),
$$

where $C, \gamma>0$ are positive constants. The one-dimensional bundle $X^{1}(t), t \in$ $\mathbb{R}$, referred to as the principal Floquet bundle, is uniquely determined; it is an analogue of the principal eigenfunction for elliptic operators. The exponential separation is a generalization of the fact that the principal eigenvalue of an elliptic operator is larger than the real part of any other eigenvalue. See $[14,16,25,26]$ for a more detailed discussion of the connection of principal Floquet bundles with principal eigenvalues and eigenfunctions.

The study of principal Floquet bundles and exponential separation in nonautonomous parabolic equations with general time-dependence originated in [21], [33]. This research was motivated by some open questions in nonlinear parabolic equations, in particular, the problem of typical asymptotic behavior in periodic-parabolic equations (see $[32,33,9]$ ). Other applications can be found in $[15,16,17,22,23,24,25,26,37]$. We also mention that in one space dimension Floquet bundles corresponding to any nodal number can be established, thus extending the classical Sturm-Liouville theorem (see $[4,5,38])$.

On unbounded domains, similar results for nonautonomous equations do not seem to be available. Of course, one does not expect the exponential separation between positive and sign-changing solutions to be valid in general (consider the Laplace operator on $\mathbb{R}^{N}$, for example). Even in the autonomous case, for the existence of a principal eigenvalue with exponential separation one needs an assumption that the "top of the spectrum" of the underlying operator be larger than the "top of the essential spectrum". In case the essential spectrum is known to be contained in the left half-plane, one needs to assume the existence of a positive eigenvalue, or equivalently, an instability condition on the corresponding semigroup. Under such additional conditions, the existence of a principal eigenvalue and eigenfunction with similar properties as on bounded domains can be proved; see [35] for classical results on autonomous operators and $[6,7]$ for extensions to time-periodic parabolic 
operators.

In this paper, we give sufficient conditions under which principal Floquet bundle exponentially separated from a complementary invariant bundle can be established for equations with general time-dependence. The conditions involve an instability assumption on (1.1) as well as a sign condition on the function $a(a \leq 0$ near $|x|=\infty)$. Note that in the time-autonomous case, the latter condition guarantees that the essential spectrum of $\Delta+a$ is in the left half-plane.

At this stage we are not attempting to prove the results in their most general form. Rather, our main goal is to understand the effects of the nonautonomous structure of the equation in combination with the unboundedness of the domain on the relative behavior of positive and sign-changing solutions. Even in the simplest example of the nonautonomous equation on $\mathbb{R}^{N}$, the extension of available results on exponential separation (for bounded domains or for autonomous or time-periodic equations on $\mathbb{R}^{N}$ ) is far from straightforward. We carry out a natural approach - obtaining the principal Floquet bundle as the limit of the principal Floquet bundles for Dirichlet problems on large balls. The key issues in this approach are uniform (radius independent) estimates of solutions on large balls, which facilitate the passage to a nontrivial limit solution and guarantee that the exponent in the separation does not shrink to zero as the balls expand.

The paper is organized as follows. In Section 2 we collect our standing hypotheses and formulate the main results. Section 3 contains reference material which will be used in the proof of the main theorems. These proofs occupy Sections 4-7. In Section 8 we give an example illustrating the importance of some of the hypotheses. Finally, in Section 9 we state our results in a more general setting of equation (1.2) and indicate the necessary modifications of the proofs.

\section{Main results}

\subsection{Standing hypotheses and some terminology}

Throughout the paper we assume that either conditions (A) and (H1) below hold simultaneously or that (A) and (H2) hold. We will specify which of these assumptions are imposed, but as we shall later show, they are equivalent.

(A) There are positive constants $d_{0}$ and $\rho_{0}$ such that $\|a\|_{L^{\infty}\left(\mathbb{R}^{N} \times \mathbb{R}\right)} \leq d_{0}$ and 


$$
a(x, t) \leq 0 \text { for almost all }(x, t) \in \mathbb{R}^{N} \times \mathbb{R} \text { with }|x| \geq \rho_{0} .
$$

To state the next assumption consider the following problem

$$
\begin{array}{llrl}
u_{t} & =\Delta u+a(x, t) u & \text { in } & B_{R} \times\left(s_{0}, \infty\right), \\
u & =0 & \text { on } \quad & \partial B_{R} \times\left(s_{0}, \infty\right),
\end{array}
$$

where $s_{0} \in \mathbb{R}, R>0$, and $B_{R}$ stands for the ball in $\mathbb{R}^{N}$ centered at the origin with radius $R$.

(H1) There exist positive constants $C_{0}, \epsilon_{0}, R_{0}$ with the following property. For each $s_{0} \in \mathbb{R}$, problem (2.1) with $R=R_{0}$ has a positive solution $u$ with initial value $u\left(\cdot, s_{0}\right) \in L^{\infty}\left(B_{R_{0}}\right)$ such that

$$
\frac{\|u(\cdot, t)\|_{L^{\infty}\left(B_{R_{0}}\right)}}{\|u(\cdot, s)\|_{L^{\infty}\left(B_{R_{0}}\right)}} \geq C_{0} e^{\epsilon_{0}(t-s)} \quad\left(t \geq s \geq s_{0}\right) .
$$

We remark that it can be proved that (H1) holds for all large $R_{0}$ if it holds for some $R_{0}$, see Lemma 4.1.

(H2) There exist positive constants $\tilde{C}_{0}, \tilde{\epsilon}_{0}$ with the following property. For each $s_{0} \in \mathbb{R}$ there exists a (possibly sign-changing) solution $\phi$ of (1.1) on $J=\left(s_{0}, \infty\right)$ such that $\phi(\cdot, t) \in L^{\infty}\left(\mathbb{R}^{N}\right)$ for $t \geq s_{0}$ and

$$
\frac{\|\phi(\cdot, t)\|_{L^{\infty}\left(\mathbb{R}^{N}\right)}}{\|\phi(\cdot, s)\|_{L^{\infty}\left(\mathbb{R}^{N}\right)}} \geq \tilde{C}_{0} e^{\tilde{\epsilon}_{0}(t-s)} \quad\left(t \geq s \geq s_{0}\right) .
$$

Hypotheses (H1) and (H2) are the "instability" conditions mentioned in the introduction; (H2) is formulated in terms of (1.1) and (H1) in terms of the approximation of (1.1) by the Dirichlet problem on a large ball. In Theorem 2.4 we will show that (H1) and (H2) are equivalent.

To simplify the exposition we will adopt the following terminology. The statement "the constant $C$ depends on the given quantities" means that $C$ is determined by (some of) the constants appearing in the conditions (A), (H1), and (H2) above. More specifically, if we assume that (A) and (H1) hold then $C$ may depend (at most) on $N, d_{0}, \rho_{0}, C_{0}, \epsilon_{0}, R_{0}$ and if we assume that (A) and (H2) holds then $C$ may depend on $N, d_{0}, \rho_{0}, \tilde{C}_{0}, \tilde{\epsilon}_{0}$.

We will frequently make use of the adjoint problem to (1.1)

$$
-v_{t}=\Delta v+a(x, t) v \quad \text { in } \quad \mathbb{R}^{N} \times J,
$$


and the adjoint problem to $(2.1)$ (with $s_{0}=-\infty$ )

$$
\begin{array}{crrr}
-v_{t} & =\Delta v+a(x, t) v & \text { in } & B_{R} \times \mathbb{R}, \\
v & =0 & \text { on } & \partial B_{R} \times \mathbb{R} .
\end{array}
$$

Replacing $t$ with $-t$ in (2.4) we obtain a new equation of the same form as (1.1). We will see below that if conditions (A) and (H1) (or (A) and (H2)) are satisfied for (1.1) then the same is true for this new equation. Thus any result which is stated for equation (1.1) (or (2.1)) can be reformulated to apply to (2.4) (or (2.5)) by replacing $t$ with $-t$. We will use this observation in the sequel, often without notice.

\subsection{Statement of the main results}

We call a solution of (1.1) (and similarly for other equations in this paper) an entire solution if it is a solution on $J=\mathbb{R}$. The first result establishes the existence of "well behaved" positive entire solutions of (1.1) and (2.4).

Theorem 2.1. Assume (A) and (H1). Then there exist positive entire solutions $\varphi, \psi$ of (1.1) and (2.4), respectively, such that for all $(x, t) \in \mathbb{R}^{N} \times \mathbb{R}$

$$
\frac{\varphi(x, t)}{\|\varphi(\cdot, t)\|_{L^{\infty}\left(\mathbb{R}^{N}\right)}} \leq c_{1} e^{-\sqrt{\epsilon_{0}}|x|}, \frac{\psi(x, t)}{\|\psi(\cdot, t)\|_{L^{\infty}\left(\mathbb{R}^{N}\right)}} \leq c_{1} e^{-\sqrt{\epsilon_{0}}|x|}
$$

and for all $t \geq s$

$$
\frac{\|\varphi(\cdot, t)\|_{L^{\infty}\left(\mathbb{R}^{N}\right)}}{\|\varphi(\cdot, s)\|_{L^{\infty}\left(\mathbb{R}^{N}\right)}} \geq c_{2} e^{\epsilon_{0}(t-s)} \quad \text { and } \quad \frac{\|\psi(\cdot, t)\|_{L^{\infty}\left(\mathbb{R}^{N}\right)}}{\|\psi(\cdot, s)\|_{L^{\infty}\left(\mathbb{R}^{N}\right)}} \leq c_{3} e^{-\epsilon_{0}(t-s)} .
$$

Here, $\epsilon_{0}$ is as in (H1) and $c_{1}, c_{2}, c_{3}$ are positive constants depending only on the given quantities.

Our main result is the following theorem on exponential separation and principal Floquet bundles. We denote by $u\left(\cdot, t ; s, u_{0}\right) \in L^{\infty}\left(\mathbb{R}^{N}\right)$ the solution of (1.1) with the initial condition $u(\cdot, s)=u_{0} \in L^{\infty}\left(\mathbb{R}^{N}\right)$.

Theorem 2.2. Assume (A) and (H1). Let $\varphi, \psi$ be as in Theorem 2.1 and let

$$
\begin{aligned}
& X^{1}(t):=\operatorname{span}\{\varphi(\cdot, t)\}, \\
& X^{2}(t):=\left\{v \in L^{\infty}\left(\mathbb{R}^{N}\right): \int_{\mathbb{R}^{N}} \psi(x, t) v(x)=0\right\} \quad(t \in \mathbb{R}) .
\end{aligned}
$$

Then the following statements hold true. 
(i) $X^{1}(t)$ and $X^{2}(t)$ are closed subspaces of $L^{\infty}\left(\mathbb{R}^{N}\right)$. They are invariant under (1.1) in the following sense: if $i \in\{1,2\}, u_{0} \in X^{i}(s)$, then $u\left(\cdot, t ; s, u_{0}\right) \in X^{i}(t)(t \geq s)$. Moreover, $X^{1}(t), X^{2}(t)$ are complementary subspaces of $L^{\infty}\left(\mathbb{R}^{N}\right)$ :

$$
L^{\infty}\left(\mathbb{R}^{N}\right)=X^{1}(t) \oplus X^{2}(t) \quad(t \in \mathbb{R}) .
$$

(ii) There are constants $C, \gamma>0$ depending only on the given quantities such that for any $u_{0} \in X^{2}(s)$ one has

$$
\frac{\left\|u\left(\cdot, t ; s, u_{0}\right)\right\|_{L^{\infty}\left(\mathbb{R}^{N}\right)}}{\|\varphi(\cdot, t)\|_{L^{\infty}\left(\mathbb{R}^{N}\right)}} \leq C e^{-\gamma(t-s)} \frac{\left\|u_{0}\right\|_{L^{\infty}\left(\mathbb{R}^{N}\right)}}{\|\varphi(\cdot, s)\|_{L^{\infty}\left(\mathbb{R}^{N}\right)}} \quad(t \geq s) .
$$

We refer to the collection of the one-dimensional spaces $X^{1}(t), t \in \mathbb{R}$, as the principal Floquet bundle of (1.1) and to $X^{2}(t), t \in \mathbb{R}$, as its complementary Floquet bundle. Property (ii) is an exponential separation between these two bundles, $C$ and $\gamma$ are referred to as the constant and exponent of the separation. As discussed in the introduction, the existence of the Floquet bundles with exponential separation extends in a natural way properties of the principal eigenvalue of time-independent (or time-periodic) parabolic problems. The positive entire solution $\varphi$ serves as an analogue of the principal eigenfunction. It is worth mentioning that its exponential spatial decay is related to the exponential decay of eigenfunctions corresponding to isolated finite-multiplicity eigenvalues of elliptic operators (cp. $[1,2,34])$.

Remark 2.3. In statement (ii), the $L^{\infty}$-norms can be replaced by $L^{2}$-norms More precisely, the following statement holds true. There are constants $\tilde{C}, \tilde{\gamma}>0$ depending only on the given quantities such that for any $u_{0} \in$ $X^{2}(s) \cap L^{2}\left(\mathbb{R}^{N}\right)$ one has

$$
\frac{\left\|u\left(\cdot, t ; s, u_{0}\right)\right\|_{L^{2}\left(\mathbb{R}^{N}\right)}}{\|\varphi(\cdot, t)\|_{L^{2}\left(\mathbb{R}^{N}\right)}} \leq \tilde{C} e^{-\tilde{\gamma}(t-s)} \frac{\left\|u_{0}\right\|_{L^{2}\left(\mathbb{R}^{N}\right)}}{\|\varphi(\cdot, s)\|_{L^{2}\left(\mathbb{R}^{N}\right)}} \quad(t \geq s) .
$$

We include the proof of this fact at the end of Section 5. Since $L^{2}\left(\mathbb{R}^{N}\right) \cap$ $L^{\infty}\left(\mathbb{R}^{N}\right)$ is dense in $L^{2}\left(\mathbb{R}^{N}\right)$, one can replace $L^{\infty}\left(\mathbb{R}^{N}\right)$ by $L^{2}\left(\mathbb{R}^{N}\right)$ in the formulation of Theorem 2.2.

Note that by Theorem 2.1 the conditions (A) and (H1) imply that (H2) is satisfied with $\tilde{C}_{0}=c_{2}$ and $\tilde{\epsilon}_{0}=\epsilon_{0}$. Our next result shows that in fact the 
conditions (H1) and (H2) are equivalent (assuming (A)). To state it let us recall that it has been proved in [16] that for any $R>0$ there exists a unique positive entire solution $\varphi_{R}$ of $(2.1)$ such that $\left\|\varphi_{R}(\cdot, 0)\right\|_{L^{\infty}\left(B_{R}\right)}=1$.

Theorem 2.4. Assume (A) and (H2). Then (H1) holds. More precisely, assume that for some $s_{0} \in \mathbb{R}$ there exists a solution $\phi$ of (1.1) satisfying (2.3). Then for each $\epsilon_{0}<\tilde{\epsilon}_{0}$ there exist $R_{0}$ and $C_{0}$ such that

$$
\frac{\left\|\varphi_{R_{0}}(\cdot, t)\right\|_{L^{\infty}\left(B_{R_{0}}\right)}}{\left\|\varphi_{R_{0}}(\cdot, s)\right\|_{L^{\infty}\left(B_{R_{0}}\right)}} \geq C_{0} e^{\epsilon_{0}(t-s)} \quad\left(t \geq s \geq s_{0}\right) .
$$

The constants $R_{0}$ and $C_{0}$ depend only on $\tilde{\epsilon}_{0}-\epsilon_{0}$ and the given quantities (which in this case are $N, d_{0}, \rho_{0}, \tilde{C}_{0}, \tilde{\epsilon}_{0}$ ). Consequently, hypothesis (H1) in Theorems 2.1 and 2.2 can be replaced by (H2).

It is natural to ask whether the positive entire solution given by Theorem 2.1 is a unique positive entire solution, up to scalar multiples. The following result gives the uniqueness in a class of localized solutions. This is hardly an optimal result, although restricting the class of admissible solutions is probably necessary for the uniqueness. Similar uniqueness problems for bounded domains have been addressed in several papers, see for example $[11,13,14,16,23,24,29]$. A different result for nonautonomous equations on time-dependent domains can be found in [28].

Proposition 2.5. Assume that (A) and (H1) or (A) and (H2) hold. Let $\varphi$ be as in Theorem 2.1 and let $\tilde{\varphi}$ be a positive entire solution of (1.1) such that $\tilde{\varphi}(\cdot, t) \in L^{\infty}\left(\mathbb{R}^{N}\right)$ for all $t$ and for some positive constants $R, k$ one has

$$
\|\tilde{\varphi}(\cdot, t)\|_{L^{\infty}\left(\mathbb{R}^{N}\right)} \leq k\|\tilde{\varphi}(\cdot, t)\|_{L^{\infty}\left(B_{R}\right)} \quad(t \in \mathbb{R}) .
$$

Then there exists a constant $q>0$ such that $\tilde{\varphi} \equiv q \varphi$ on $\mathbb{R}^{N} \times \mathbb{R}$. An analogous conclusion holds for positive entire solutions of (2.4).

Theorem 2.4 can be interpreted as a robustness of the instability condition when $\mathbb{R}^{N}$ is replaced by a large ball. There are many other robustness results that one can address in connection with exponential separations, see [16] for an account of such results for bounded Lipschitz domains. Many of these results can be carried over to the problem on $\mathbb{R}^{N}$ using the methods of the present paper. To keep the length of the paper within reasonable limits, we only prove one of the most basic results, the robustness of the instability 
condition (and hence the existence of the principal Floquet bundle and exponential separation) for equations with a perturbed coefficient $a$. We thus consider the problem

$$
u_{t}=\Delta u+\tilde{a}(x, t) u \quad \text { in } \quad \mathbb{R}^{N} \times J
$$

with $\tilde{a} \in L^{\infty}\left(\mathbb{R}^{N} \times \mathbb{R}\right)$.

Theorem 2.6. Assume (A) and (H2). Then (H2) also holds for (2.13) provided $\|a-\tilde{a}\|_{L^{\infty}\left(\mathbb{R}^{N} \times \mathbb{R}\right)}$ is sufficiently small. More precisely, for each $\epsilon<\tilde{\epsilon}_{0}$ there exist positive constants $\delta, \tilde{c}_{3}, \tilde{c}_{4}$ depending only on $N, d_{0}, \rho_{0}, \tilde{C}_{0}, \tilde{\epsilon}_{0}$ and $\tilde{\epsilon}_{0}-\epsilon$ such that if $\tilde{a} \in L^{\infty}\left(\mathbb{R}^{N} \times \mathbb{R}\right)$ and $\|a-\tilde{a}\|_{L^{\infty}\left(\mathbb{R}^{N} \times \mathbb{R}\right)}<\delta$ then equation (2.13) has a positive entire solution $\tilde{\varphi}$ satisfying

$$
\begin{aligned}
& \frac{\tilde{\varphi}(x, t)}{\|\tilde{\varphi}(\cdot, t)\|_{L^{\infty}\left(\mathbb{R}^{N}\right)}} \leq \tilde{c}_{3} e^{-\sqrt{\epsilon}|x|} \quad\left(x \in \mathbb{R}^{N}, t \in \mathbb{R}\right) \\
& \frac{\|\tilde{\varphi}(\cdot, t)\|_{L^{\infty}\left(\mathbb{R}^{N}\right)}}{\|\tilde{\varphi}(\cdot, s)\|_{L^{\infty}\left(\mathbb{R}^{N}\right)}} \geq \tilde{c}_{4} e^{\epsilon(t-s)} \quad(-\infty<s<t<\infty) .
\end{aligned}
$$

Remark 2.7. (i) If $\epsilon$ is close to $\tilde{\epsilon}_{0}$ and $\delta$ is chosen small enough, specifically, if $\delta<\tilde{\epsilon}_{0} / 2<\epsilon$, then (2.15) and Theorem 2.4 imply the existence of an exponential separation for (2.13) with constant $\tilde{C}$ and exponent $\tilde{\gamma}$ depending only on the indicated quantities. Indeed, to apply Theorem 2.4 , one first uses the transformation $u \rightarrow e^{-\delta t} u$. This produces the coefficient $\tilde{a}-\delta$, which is negative outside $B_{\rho_{0}}$ (by $(\mathrm{A})$ and the condition $\left.\|a-\tilde{a}\|_{L^{\infty}\left(\mathbb{R}^{N} \times \mathbb{R}\right)}<\delta\right)$ and the transformed positive entire solution $\varphi(\cdot, t) e^{-\delta t}$ still has an exponential growth.

(ii) Once (H2) holds for some exponent $\tilde{\epsilon}_{0}>0$, so that an entire positive solution $\varphi$ is given by Theorems 2.4 and 2.2 , the maximal exponential growth in (H2) is achieved by taking $\phi=\varphi$. In particular, we can choose $\tilde{\epsilon}_{0}$ arbitrarily close to (but smaller than) the exponent

$$
\underline{\lambda}(\varphi):=\liminf _{t-s \rightarrow \infty} \frac{\ln \|\varphi(\cdot, t)\|_{L^{\infty}\left(\mathbb{R}^{N}\right)}-\ln \|\varphi(\cdot, s)\|_{L^{\infty}\left(\mathbb{R}^{N}\right)}}{t-s},
$$

usually referred to as the lower singular exponent (or sometimes, the lower Lyapunov exponent) of $\varphi$. 


\section{$3 \quad$ Preliminary results}

In this section we collect results that will be used frequently throughout the paper. First, we state the interior Harnack inequality $([3,27])$.

Theorem 3.1. Given a domain $\Omega \subseteq \mathbb{R}^{N}$, let $v$ be a nonnegative solution of $u_{t}=\Delta u+a(x, t) u$ on $\Omega \times(0, \infty)$. Assume that $0<\delta<T, d>0$, and $D$ is a bounded subdomain of $\Omega$ such that $\operatorname{dist}(\bar{D}, \partial \Omega) \geq d$. Then there is a positive constant $C$ depending only on $\delta, T, D, d$ and the given quantities such that

$$
\sup _{x \in D} v(x, s) \leq C \inf _{x \in D} v(x, t)
$$

for each $t, s \in \mathbb{R}$ such that $s \geq \delta^{2}$ and $T \geq t-s \geq \delta^{2}$.

The next result is a generalization of the previous theorem; it has been proved in a more general form for supersolutions of second order linear parabolic equations in [30, Lemma 3.5]. Here, we state it in a simplified form tailored to our needs. In the following text we denote by $f^{+}\left(f^{-}\right)$the positive (negative) part of a real valued function $f$.

Theorem 3.2. Given $R>0, \tau<\tau_{1}<\tau_{2}<\tau_{3}<\tau_{4}$, there is a positive constant $\kappa$ determined only by $R, \tau_{1}-\tau, \tau_{2}-\tau_{1}, \tau_{3}-\tau_{2}, \tau_{4}-\tau_{3}$, and the given quantities such that the following holds. Let $u \in C\left(\bar{B}_{R+1} \times[\tau, \infty)\right)$ be a solution of $u_{t}=\Delta u+a(x, t) u$ on $B_{R+1} \times(\tau, \infty), \tau \in \mathbb{R}$. Then

$$
\begin{aligned}
u(x, t) \geq \kappa \| u^{+} & \left\|_{L^{\infty}\left(B_{R} \times\left(\tau_{1}, \tau_{2}\right)\right)}-e^{d_{0}\left(\tau_{4}-\tau\right)}\right\| u^{-} \|_{L^{\infty}\left(B_{R+1} \times\left(\tau, \tau_{4}\right)\right)}, \\
& \left((x, t) \in B_{R} \times\left(\tau_{3}, \tau_{4}\right)\right) .
\end{aligned}
$$

Consider now the following initial value problem

$$
\begin{aligned}
u_{t} & =\Delta u+a(x, t) u & & \text { in } \quad \mathbb{R}^{N} \times(0, \infty), \\
u(x, 0) & =u_{0}(x) & & x \in \mathbb{R}^{N},
\end{aligned}
$$

with $u_{0} \in L^{\infty}\left(\mathbb{R}^{N}\right)$. The unique solution $u(\cdot, t) \in L^{\infty}\left(\mathbb{R}^{N}\right)$ of $(3.3)$ has the integral representation

$$
u(x, t)=\int_{\mathbb{R}^{N}} \Gamma(x, t ; y, 0) u_{0}(y) d y \quad\left((x, t) \in \mathbb{R}^{N} \times(0, \infty)\right),
$$


where $\Gamma(x, t ; y, s)$ is the weak Green's function [3]. We refer to [3, Theorem 10] for basic properties of $\Gamma(\cdot, \cdot ; \cdot, \cdot)$. In particular, a simple comparison argument with the heat kernel shows that for any $t>s, s \in \mathbb{R}$, it satisfies the following inequality

$0<\Gamma(x, t ; y, s) \leq e^{d_{0}(t-s)}(4 \pi(t-s))^{-N / 2} e^{-|x-y|^{2} / 4(t-s)} \quad\left((x, y) \in \mathbb{R}^{N} \times \mathbb{R}^{N}\right)$,

where $d_{0}$ is as in (A). As a consequence we obtain the following lemma.

Lemma 3.3. Let $u$ be a solution of (3.3) with $u_{0} \in L^{\infty}\left(\mathbb{R}^{N}\right)$ such that $u_{0}(x)=0$ for $\left|x-x_{0}\right| \leq \sigma$, where $x_{0} \in \mathbb{R}^{N}$ and $\sigma>0$ are fixed. Then for any $t>0$ we have

$$
\left|u\left(x_{0}, t\right)\right| \leq \frac{c(N) t^{N / 2} e^{d_{0} t}}{\sigma^{N}}\left\|u_{0}\right\|_{L^{\infty}\left(\mathbb{R}^{N}\right)},
$$

where $c(N)$ depends only on $N$.

Proof. First an elementary computation for $\beta>0$ shows that

$$
\int_{|\xi| \geq \beta} e^{-|\xi|^{2}} d \xi \leq \tilde{c}(N) \beta^{-N}
$$

where $\tilde{c}(N)>0$ depends only on $N$.

Suppose $t>0$. Using (3.4), (3.5), and our assumption on $u_{0}$ we obtain

$$
\left|u\left(x_{0}, t\right)\right| \leq\left(\int_{\left|\xi-x_{0}\right| \geq \sigma} e^{d_{0} t}(4 \pi t)^{-N / 2} e^{-\left|x_{0}-\xi\right|^{2} / 4 t} d \xi\right)\left\|u_{0}\right\|_{L^{\infty}\left(\mathbb{R}^{N}\right)} .
$$

Substituting $\eta=\left(\xi-x_{0}\right) / \sqrt{4 t}$ in the integral above and using (3.7), we conclude

$$
\left|u\left(x_{0}, t\right)\right| \leq \frac{\tilde{c}(N) 2^{N} t^{N / 2} e^{d_{0} t}}{\pi^{N / 2} \sigma^{N}}\left\|u_{0}\right\|_{L^{\infty}\left(\mathbb{R}^{N}\right)},
$$

which implies (3.6) with $c(N)=\tilde{c}(N) 2^{N} / \pi^{N / 2}$.

The following result is an immediate consequence of the standard maximum principle $([18])$.

Proposition 3.4. Let $u$ be a solution of (3.3) with $u_{0} \in L^{\infty}\left(\mathbb{R}^{N}\right)$. Then

$$
\|u(t)\|_{L^{\infty}\left(\mathbb{R}^{N}\right)} \leq e^{d_{0} t}\left\|u_{0}\right\|_{L^{\infty}\left(\mathbb{R}^{N}\right)} \quad(t \geq 0) .
$$

Replacing $\mathbb{R}^{N}$ by $B_{R}$, the same estimate holds for solutions of the initial value problem associated with (2.1). 
As in this proposition, we often shorten the notation and suppress the argument $x$ of solutions; thus we write $u(t)$ or $u(\cdot, t)$ with the same meaning.

Next, we state another version of the maximum principle.

Lemma 3.5. Suppose (A) holds. Let $u$ be a solution of (3.3) with $u_{0} \in$ $L^{\infty}\left(\mathbb{R}^{N}\right) \cap C\left(\mathbb{R}^{N}\right)$. Then for all $t>0$ we have

$$
\sup _{\substack{|x| \geq \rho_{0} \\ s \in[0, t]}} u(x, s) \leq \max \left\{\sup _{|x| \geq \rho_{0}} u_{0}(x) ; \sup _{\substack{|x|=\rho_{0} \\ s \in[0, t]}} u(x, s)\right\}
$$

Proof. Denote the right hand side in (3.9) by $M$. Then the condition (A) guarantees that $u-M$ is a bounded (continuous up to the boundary) subsolution of (1.1) on $\left(\mathbb{R}^{N} \backslash \bar{B}_{\rho_{0}}\right) \times(0, t)$, which is nonpositive on the parabolic boundary of that set. If the solution $u$ were classical then [18, Theorem 8.1.4] would guarantee that $u \leq M$ on $\left(\mathbb{R}^{N} \backslash \bar{B}_{\rho_{0}}\right) \times(0, t)$. Even though $u$ may not be classical, since we only assume that $a$ is bounded, due to the more general version of the maximum principle for strong solutions [20, Theorem 7.1], the proof of [18, Theorem 8.1.4] also works in our more general setting. Therefore $u \leq M$ on $\left(\mathbb{R}^{N} \backslash \bar{B}_{\rho_{0}}\right) \times(0, t)$, as claimed.

For future use we formulate here the following corollary which readily follows from Lemma 3.5 and a continuity argument. Note that in this corollary we only assume $u_{0} \in L^{\infty}\left(\mathbb{R}^{N}\right)$ but of course $u(\cdot, t) \in L^{\infty}\left(\mathbb{R}^{N}\right) \cap C\left(\mathbb{R}^{N}\right)$ for all positive times.

Corollary 3.6. Let $u$ be the solution of (3.3) with $u_{0} \in L^{\infty}\left(\mathbb{R}^{N}\right)$ and $u_{0} \geq 0$. Assume that $\|u(\cdot, t)\|_{L^{\infty}\left(\mathbb{R}^{N}\right)} \geq b>\left\|u_{0}\right\|_{L^{\infty}\left(\mathbb{R}^{N}\right)}$ for some $b, t>0$. Then there exists $t_{0} \in(0, t]$ such that $\left\|u\left(\cdot, t_{0}\right)\right\|_{L^{\infty}\left(\mathbb{R}^{N}\right)}=\left\|u\left(\cdot, t_{0}\right)\right\|_{L^{\infty}\left(B_{\rho_{0}}\right)} \geq b$.

Remark 3.7. (i) Obviously, there is nothing special about the interval $(0, \infty)$ in the above results. These results hold in the same way on any interval $(s, \infty)$ with $s \in \mathbb{R}$.

(ii) Lemma 3.5 and and Corollary 3.6 apply equally well to solutions of the initial value problem associated with (2.1).

\section{$4 \quad$ Proof of Theorem 2.1}

Throughout the section we assume that hypotheses (A) and (H1) are satisfied. We first collect some known properties of positive entire solutions of the 
homogeneous Dirichlet problem on bounded domains. For $R \geq \rho_{0}+R_{0}+1$ ( $\rho_{0}$ as in $(\mathrm{A})$ and $R_{0}$ as in (H1)) consider the problems

$$
\begin{array}{llr}
u_{t}=\Delta u+a(x, t) u & \text { in } \quad B_{R} \times \mathbb{R}, \\
u=0 & \text { on } \quad \partial B_{R} \times \mathbb{R},
\end{array}
$$

and

$$
\begin{aligned}
& -v_{t}=\Delta v+a(x, t) v \quad \text { in } \quad B_{R} \times \mathbb{R}, \\
& v=0 \quad \text { on } \quad \partial B_{R} \times \mathbb{R} \text {. }
\end{aligned}
$$

It is proved in [16] that there are unique positive entire solutions $\varphi_{R}, \psi_{R}$ of (4.1) and (4.2), respectively, with $\left\|\varphi_{R}(\cdot, 0)\right\|_{L^{\infty}\left(B_{R}\right)}=\left\|\psi_{R}(\cdot, 0)\right\|_{L^{\infty}\left(B_{R}\right)}=1$. Moreover, by [16, Lemma 3.9] one has

$$
\left\|\varphi_{R}(\cdot, t)\right\|_{L^{\infty}\left(B_{R}\right)} \leq C(R) \inf _{x \in B_{R_{0}}} \varphi_{R}(x, t) \quad(t \in \mathbb{R}),
$$

where $C(R)>0$ depends only on $R$ and the given quantities, and the same estimate holds for $\psi_{R}$.

We want to prove the existence of entire solutions of (1.1) and (2.4) by taking the limits of $\varphi_{R}$ and $\psi_{R}$ as $R \rightarrow \infty$. We prepare the limiting procedure by a sequence of lemmas in which we prove that $\varphi_{R}$ and $\psi_{R}$ have properties similar to those stated in Theorem 2.1 for $\varphi$ and $\psi$.

We start by proving that the growth of $u$ in (2.2) forces a similar growth of $\varphi_{R}$ for all $R>\rho_{0}+R_{0}+1$ (independently of $R$ ).

Lemma 4.1. There is a positive constant $c_{6}$ depending only on the given quantities such that for all $R \geq \rho_{0}+R_{0}+1$ one has

$$
\frac{\left\|\varphi_{R}(\cdot, t)\right\|_{L^{\infty}\left(B_{R}\right)}}{\left\|\varphi_{R}(\cdot, s)\right\|_{L^{\infty}\left(B_{R}\right)}} \geq c_{6} e^{\epsilon_{0}(t-s)} \quad(t \geq s)
$$

where $\epsilon_{0}$ is as in (H1).

Proof. We first prove that estimate (4.4) holds with $\epsilon_{0}$ replaced with some $\epsilon_{1}$. Take the solution $u$ as in (H1). We will repeatedly use a comparison between (multiples of) $u$ and $\varphi_{R}$, with $R>R_{0}$. This is legitimate (on $B_{R_{0}}$ ) as they solve the same equation and $\varphi_{R}>0=u$ on $\partial B_{R_{0}}$.

Fix $\tilde{t} \in \mathbb{R}$. By (4.3) we have

$$
\frac{C(R) \varphi_{R}(x, \tilde{t})}{\left\|\varphi_{R}(\cdot, \tilde{t})\right\|_{L^{\infty}\left(B_{R}\right)}} \geq 1 \geq \frac{u(x, \tilde{t})}{\|u(\cdot, \tilde{t})\|_{L^{\infty}\left(B_{R_{0}}\right)}} \quad\left(x \in B_{R_{0}}\right) .
$$


This implies, by comparison and (2.2), that for $\tau_{R}=\left(1 / \epsilon_{0}\right) \ln \left(2 C(R) / C_{0}\right)$ we have

$$
\frac{C(R)\left\|\varphi_{R}\left(\cdot, \tilde{t}+\tau_{R}\right)\right\|_{L^{\infty}\left(B_{R}\right)}}{\left\|\varphi_{R}(\cdot, \tilde{t})\right\|_{L^{\infty}\left(B_{R}\right)}} \geq \frac{\left\|u\left(\cdot, \tilde{t}+\tau_{R}\right)\right\|_{L^{\infty}\left(B_{R_{0}}\right)}}{\|u(\cdot, \tilde{t})\|_{L^{\infty}\left(B_{R_{0}}\right)}} \geq C_{0} e^{\epsilon_{0} \tau_{R}}=2 C(R) .
$$

Hence $\left\|\varphi_{R}\left(\cdot, \tilde{t}+\tau_{R}\right)\right\|_{L^{\infty}\left(B_{R}\right)} \geq 2\left\|\varphi_{R}(\cdot, \tilde{t})\right\|_{L^{\infty}\left(B_{R}\right)}$. By Corollary 3.6 (cf. Remark 3.7(ii)), there exists $t_{0} \in\left(\tilde{t}, \tilde{t}+\tau_{R}\right]$ such that

$$
\left\|\varphi_{R}\left(\cdot, t_{0}\right)\right\|_{L^{\infty}\left(B_{R}\right)}=\left\|\varphi_{R}\left(\cdot, t_{0}\right)\right\|_{L^{\infty}\left(B_{\rho_{0}}\right)} \geq 2\left\|\varphi_{R}(\cdot, \tilde{t})\right\|_{L^{\infty}\left(B_{R}\right)} .
$$

The Harnack inequality (3.1) then implies

$$
\left\|\varphi_{R}\left(\cdot, t_{0}\right)\right\|_{L^{\infty}\left(B_{R}\right)}=\left\|\varphi_{R}\left(\cdot, t_{0}\right)\right\|_{L^{\infty}\left(B_{\rho_{0}}\right)} \leq C\left(\rho_{0}\right) \inf _{x \in B_{R_{0}}} \varphi_{R}\left(x, t_{0}+1\right),
$$

where $C\left(\rho_{0}\right)>1$ is independent of $R$. By Proposition 3.4, we also have $\left\|\varphi_{R}\left(\cdot, t_{0}+1\right)\right\|_{L^{\infty}\left(B_{R}\right)} \leq e^{d_{0}}\left\|\varphi_{R}\left(\cdot, t_{0}\right)\right\|_{L^{\infty}\left(B_{R}\right)}$. Combining this fact with (4.7), we obtain

$$
\left\|\varphi_{R}\left(\cdot, t_{0}+1\right)\right\|_{L^{\infty}\left(B_{R}\right)} \leq \tilde{C} \inf _{x \in B_{R_{0}}} \varphi_{R}\left(x, t_{0}+1\right),
$$

where $\tilde{C}=C\left(\rho_{0}\right) e^{d_{0}}$ is independent of $R$. Thus (4.5) holds with $C(R)$ replaced by $\tilde{C}$ and $\tilde{t}$ replaced by $t_{0}+1$. We now take $\tau=\left(1 / \epsilon_{0}\right) \ln \left(2 C\left(\rho_{0}\right) \tilde{C} / C_{0}\right)$ (with $C\left(\rho_{0}\right)$ as in (4.7)) and obtain, estimating as in (4.6), that

$$
\left\|\varphi_{R}\left(\cdot, t_{0}+1+\tau\right)\right\|_{L^{\infty}\left(B_{R}\right)} \geq 2 C\left(\rho_{0}\right)\left\|\varphi_{R}\left(\cdot, t_{0}+1\right)\right\|_{L^{\infty}\left(B_{R}\right)} .
$$

Arguing as above, we consequently establish the existence of $t_{1} \in\left(t_{0}+1, t_{0}+\right.$ $1+\tau]$ such that $\left\|\varphi_{R}\left(\cdot, t_{1}\right)\right\|_{L^{\infty}\left(B_{R}\right)}=\left\|\varphi_{R}\left(\cdot, t_{1}\right)\right\|_{L^{\infty}\left(B_{\rho_{0}}\right)}$ and

$$
\left\|\varphi_{R}\left(\cdot, t_{1}\right)\right\|_{L^{\infty}\left(B_{R}\right)} \geq 2 C\left(\rho_{0}\right)\left\|\varphi_{R}\left(\cdot, t_{0}+1\right)\right\|_{L^{\infty}\left(B_{R}\right)} \geq 2\left\|\varphi_{R}\left(\cdot, t_{0}\right)\right\|_{L^{\infty}\left(B_{R}\right)},
$$

where we used (4.7) in the last inequality. We can repeat this process (from now on $\tau$ stays fixed) indefinitely to find a sequence $\left\{t_{i}\right\}_{i=0}^{\infty}$ with the following properties. For each $i \geq 0$, we have $t_{i+1} \in\left(t_{i}+1, t_{i}+1+\tau\right]$, $\left\|\varphi_{R}\left(\cdot, t_{i}\right)\right\|_{L^{\infty}\left(B_{R}\right)}=\left\|\varphi_{R}\left(\cdot, t_{i}\right)\right\|_{L^{\infty}\left(B_{\rho_{0}}\right)}$ and

$$
\left\|\varphi_{R}\left(\cdot, t_{i+1}\right)\right\|_{L^{\infty}\left(B_{R}\right)} \geq 2\left\|\varphi_{R}\left(\cdot, t_{i}\right)\right\|_{L^{\infty}\left(B_{R}\right)} .
$$

By the maximum principle (Proposition 3.4), we also have

$$
\left\|\varphi_{R}(\cdot, t)\right\|_{L^{\infty}\left(B_{R}\right)} \geq c(\tau)\left\|\varphi_{R}\left(\cdot, t_{i+1}\right)\right\|_{L^{\infty}\left(B_{R}\right)} \quad\left(t \in\left[t_{i}, t_{i+1}\right]\right) .
$$


This and (4.8) imply that estimate (4.4) holds for any $t \geq s \geq t_{0}$ and some positive constant $c_{6}$ and some exponent $\epsilon_{1} \in\left(0, \epsilon_{0}\right]$ replacing $\epsilon_{0}$. The constants $c_{6}$ and $\epsilon_{1}$ depend only on the given quantities. Since $t_{0} \in\left(\tilde{t}, \tilde{t}+\tau_{R}\right]$, the estimate holds for any $t \geq s \geq \tilde{t}+\tau_{R}$ and, since there was no restriction on $\tilde{t}$, it holds for any $t \geq s$.

We still need to show that (4.4) holds with the specific exponent $\epsilon_{0}$ (and a possibly larger $c_{6}$ ). We first claim that inequality (4.5) still holds for each $\tilde{t}$ if the constant $C(R)$ is replaced by some constant $c_{7}^{-1}>0$ depending only on the given quantities. If this is true then we are done, for replacing in (4.6) $\tilde{t}$ by $s$ and $\tau_{R}$ by $t-s$ we get (4.4) with $c_{6}=c_{7} C_{0}$. Let us now prove the claim. Using (4.4) (with $\epsilon_{0}$ replaced by $\epsilon_{1}$ ) we find $\tau$, determined by $c_{6}$ and $\epsilon_{1}$ such that

$$
\left\|\varphi_{R}(\cdot, \tilde{t}+\tau)\right\|_{L^{\infty}\left(B_{R}\right)}>\left\|\varphi_{R}(\cdot, \tilde{t})\right\|_{L^{\infty}\left(B_{R}\right)} \quad(\tilde{t} \in \mathbb{R}) .
$$

We then use similar arguments as at the beginning of the proof. By Corollary 3.6 (cf. Remark 3.7(ii)), (4.9) implies that for each $\tilde{t} \in \mathbb{R}$ there exists $s \in[\tilde{t}, \tilde{t}+\tau]$ such that $\left\|\varphi_{R}(\cdot, s)\right\|_{L^{\infty}\left(B_{R}\right)}=\left\|\varphi_{R}(\cdot, s)\right\|_{L^{\infty}\left(B_{\rho_{0}}\right)}$. Then, by the Harnack inequality and Proposition 3.4, we have for $t=\tilde{t}+2 \tau$ and $x \in B_{R_{0}}$

$$
\varphi_{R}(x, t) \geq c_{1}\left\|\varphi_{R}(\cdot, s)\right\|_{L^{\infty}\left(B_{\rho_{0}}\right)}=c_{1}\left\|\varphi_{R}(\cdot, s)\right\|_{L^{\infty}\left(B_{R}\right)} \geq c_{7}\left\|\varphi_{R}(\cdot, t)\right\|_{L^{\infty}\left(B_{R}\right)},
$$

where $c_{1}$ and $c_{7}$ are positive constants determined by the given quantities. Since $\tilde{t}$ is arbitrary, this implies the desired estimate. The proof of the lemma is now complete.

For future use, we display here the following property established in the proof of Lemma 4.1.

Corollary 4.2. There exists $c_{7}>0$ depending only on the given quantities such that for all $R \geq \rho_{0}+R_{0}+1$

$$
\frac{\inf _{x \in B_{R_{0}}} \varphi_{R}(x, t)}{\left\|\varphi_{R}(\cdot, t)\right\|_{L^{\infty}\left(B_{R}\right)}} \geq c_{7} \quad(t \in \mathbb{R}) .
$$

Let us remark that by [16, Lemma 3.9 and (7.2)]

$c_{8}^{-1} \leq\left\|\varphi_{\rho_{0}+R_{0}+1}(\cdot, t)\right\|_{L^{\infty}\left(B_{\rho_{0}+R_{0}+1}\right)}\left\|\psi_{\rho_{0}+R_{0}+1}(\cdot, t)\right\|_{L^{\infty}\left(B_{\rho_{0}+R_{0}+1}\right)} \leq c_{8} \quad(t \in \mathbb{R})$, 
where $c_{8}>0$ depends only on the given quantities. Obviously, inequalities (4.11) and (4.4) (with $R=\rho_{0}+R_{0}+1$ ) imply

$$
\frac{\left\|\psi_{\rho_{0}+R_{0}+1}(\cdot, t)\right\|_{L^{\infty}\left(B_{\rho_{0}+R_{0}+1}\right)}}{\left\|\psi_{\rho_{0}+R_{0}+1}(\cdot, s)\right\|_{L^{\infty}\left(B_{\rho_{0}+R_{0}+1}\right)}} \leq c_{8}^{2} c_{6}^{-1} e^{-\epsilon_{0}(t-s)} \quad(t \geq s) .
$$

In view of (4.12), the above arguments adapted to the adjoint problem (4.2) give the following lemma.

Lemma 4.3. There exists a positive constant $c_{9}$ such that for all $R \geq \rho_{0}+$ $R_{0}+2$ the following inequality holds

$$
\frac{\left\|\psi_{R}(\cdot, t)\right\|_{L^{\infty}\left(B_{R}\right)}}{\left\|\psi_{R}(\cdot, s)\right\|_{L^{\infty}\left(B_{R}\right)}} \leq c_{9} e^{-\epsilon_{0}(t-s)} \quad(t \geq s)
$$

where $\epsilon_{0}$ is as in (H1) and $c_{9}$ depends only on the given quantities. Moreover, estimate (4.10) also holds with $\varphi_{R}$ replaced by $\psi_{R}$.

Estimates (4.4) and (4.13) will enable us to prove (2.7) for a suitable limit $\varphi$ of $\varphi_{R}$. We now prove a spatial exponential decay estimate for $\varphi_{R}$ $\left(\psi_{R}\right)$ which will enable us to prove (2.6).

Lemma 4.4. For any $R \geq \rho_{0}+R_{0}+2$ the following inequality is satisfied

$$
\frac{\varphi_{R}(x, t)}{\left\|\varphi_{R}(\cdot, t)\right\|_{L^{\infty}\left(B_{R}\right)}} \leq\left(e^{\sqrt{\epsilon_{0}} \rho_{0}} / c_{6}\right) e^{-\sqrt{\epsilon_{0}}|x|} \quad\left((x, t) \in B_{R} \times \mathbb{R}\right)
$$

where $c_{6}$ and $\epsilon_{0}$ are as in Lemma 4.1. The same estimate holds if $\varphi_{R}$ is replaced by $\psi_{R}$ and $c_{6}$ is replaced by $c_{9}^{-1}$, with $c_{9}$ as in Lemma 4.3.

Proof. Replacing $\varphi_{R}(x, t)$ by $e^{-\epsilon_{0} t} \varphi_{R}(x, t)$ (which does not affect (4.14)), we can assume for a while that $a(x, t) \leq-\epsilon_{0}<0$ for $(x, t) \in \mathbb{R}^{N} \times \mathbb{R}$ with $|x| \geq \rho_{0}$. Given any $t_{0}<t$ we define

$$
\zeta_{R}(x, s)=q e^{-\epsilon_{0}\left(s-t_{0}\right)}+q e^{-\sqrt{\epsilon_{0}}\left(|x|-\rho_{0}\right)} \quad\left(x \in \mathbb{R}^{N}, s \in\left[t_{0}, t\right]\right),
$$

where $q=\left(1 / c_{6}\right)\left\|\varphi_{R}(\cdot, t)\right\|_{L^{\infty}\left(B_{R}\right)}$ and $c_{6}$ is as in Lemma 4.1. It is a routine calculation (similar to that in [31, Proof of Lemma 2.4]) to verify that for each $t_{0}<t$ the function $\zeta_{R}$ is a supersolution of (4.1) on the set $Q=\{(x, s) \in$ $\left.\left(B_{R} \backslash B_{\rho_{0}}\right) \times\left(t_{0}, t\right)\right\}$ dominating $\varphi_{R}$ on the parabolic boundary of $Q$. By the maximum principle, $\zeta_{R}$ then dominates $\varphi_{R}$ on all of $Q$, in particular, 
$\zeta_{R}(x, t) \geq \varphi_{R}(x, t)$ for all $x \in B_{R} \backslash B_{\rho_{0}}$. Sending $t_{0} \rightarrow-\infty$ we obtain $\varphi_{R}(x, t) \leq q e^{-\sqrt{\epsilon_{0}}\left(|x|-\rho_{0}\right)}$ for all $x \in B_{R} \backslash B_{\rho_{0}}$. By the definition of $q$ and since $c_{6} \leq 1$ (take $t=s$ in (4.4)), the same estimate is trivially satisfied for $x \in B_{\rho_{0}}$. This proves the lemma.

We are now ready for the proof of Theorem 2.1.

Proof of Theorem 2.1. Let $R_{n} \rightarrow \infty$ as $n \rightarrow \infty$ and let $\varphi_{R_{n}}$ be the unique positive entire solution of (4.1) with $R=R_{n}$ satisfying $\left\|\varphi_{R_{n}}(\cdot, 0)\right\|_{L^{\infty}\left(B_{R_{n}}\right)}=$ 1. Corollary 4.2, Harnack inequality, and the maximum principle imply that for some $c>0$ we have $\left\|\varphi_{R_{n}}(\cdot, t)\right\|_{L^{\infty}\left(B_{R_{n}}\right)} \leq c e^{c|t|}$ for all $t \in \mathbb{R}$ and all $n \in \mathbb{N}$. More specifically, for $t \geq 0$ this is a direct consequence of Proposition 3.4. To prove it for $t \leq 0$, one combines the maximum principle with (3.1) and (4.10). We next use a standard limiting argument (see [3], for example) to find a limit of $\varphi_{R_{n}}$ in $C_{l o c}\left(\mathbb{R}^{N} \times \mathbb{R}\right)$. It goes as follows. The bound on $\varphi_{R_{n}}$ just established and parabolic interior estimates imply that on any fixed compact subset $K$ of $\mathbb{R}^{N} \times \mathbb{R}$ the functions $\varphi_{R_{n}}$ with $n$ sufficiently large are Hölder continuous and have a Hölder norm bounded by a constant independent of $n$. Consequently, passing to a subsequence if necessary, $\varphi_{R_{n}}(\cdot, \cdot)$ converges locally uniformly in $\mathbb{R}^{N} \times \mathbb{R}$ to a nonnegative solution $\varphi$ of (1.1). Corollary 4.2 and the normalization assumption on $\varphi_{R_{n}}$ imply that $\varphi$ is nontrivial and hence (by Harnack inequality) positive on $\mathbb{R}^{N} \times \mathbb{R}$. Moreover, Lemma 4.4 shows that the convergence of $\varphi_{R_{n}}(\cdot, \cdot)$ to $\varphi$ is uniform on $\mathbb{R}^{N} \times[-t, t]$ for all $t>0$. Using this fact, Lemma 4.4, and Lemma 4.1, one immediately obtains (2.6) and (2.7) for $\varphi$. The same arguments apply equally well to $\psi_{R_{n}}$ and we establish the existence of a positive entire solution $\psi$ of (2.4) with the properties as stated in Theorem 2.1. The proof is thus complete.

\section{Proof of Theorem 2.2}

Let $\varphi$ and $\psi$ be as in Theorem 2.1. The proof of statement (i) uses the following identity for solutions of (1.1) and its adjoint equation (2.4).

Lemma 5.1. Let $a<b$. Let $u$ be a solution of $(1.1)$ on $\mathbb{R}^{N} \times(a, \infty)$ with $u(\cdot, a) \in L^{\infty}\left(\mathbb{R}^{N}\right)$ and let $v$ be a solution of $(2.4)$ on $\mathbb{R}^{N} \times(-\infty, b)$ with $v(\cdot, b) \in L^{\infty}\left(\mathbb{R}^{N}\right)$. Then

$$
\langle u(\cdot, t), v(\cdot, t)\rangle:=\int_{\mathbb{R}^{N}} u(x, t) v(x, t) d x \equiv \text { const. } \quad(t \in[a, b]),
$$


provided one of the functions $u(\cdot, t), v(\cdot, t)$ is in $L^{1}\left(\mathbb{R}^{N}\right)$ for each $t \in[a, b]$.

Proof. Suppose, for example, that $v(\cdot, t)$ is in $L^{1}\left(\mathbb{R}^{N}\right)$ for each $t \in[a, b]$. Using (3.4), the Fubini theorem, and [3, Theorem 10], we can compute for any $t \in(a, b]$

$$
\begin{aligned}
\int_{\mathbb{R}^{N}} u(x, t) v(x, t) d x & =\int_{\mathbb{R}^{N}} \int_{\mathbb{R}^{N}} \Gamma(x, t ; \xi, a) u(\xi, a) v(x, t) d \xi d x \\
& =\int_{\mathbb{R}^{N}} u(\xi, a)\left(\int_{\mathbb{R}^{N}} \Gamma(x, t ; \xi, a) v(x, t) d x\right) d \xi \\
& =\int_{\mathbb{R}^{N}} u(\xi, a) v(\xi, a) d \xi,
\end{aligned}
$$

completing the proof.

Proof of Theorem 2.2(i). The fact that $X^{i}(t), i=1,2$, are closed subspaces of $L^{\infty}\left(\mathbb{R}^{N}\right)$ is clear. The invariance of $X^{2}(t), t \in \mathbb{R}$, as stated in (i), follows from (5.1). The invariance of $X^{1}(t), t \in \mathbb{R}$, is obvious. Since $\psi(t)>0$, the space $X^{2}(t)$ contains no (nontrivial) nonnegative function. On the other hand, $X^{1}(t)$ is spanned by a positive function, hence $X^{1}(t) \cap X^{2}(t)=\{0\}$ $(t \in \mathbb{R})$. This in conjunction with a dimension-codimension count yields $(2.8)$.

The next result follows from Lemma 5.1 and the maximum principle. The details of the proof are the same as for bounded domains [14, 29] and are therefore omitted.

Lemma 5.2. Let $u$ be a solution of (3.3) with $u_{0} \in L^{\infty}\left(\mathbb{R}^{N}\right)$ such that $\left\langle u_{0}, \psi(0)\right\rangle=0$. Then $\xi(t):=\langle|u(t)|, \psi(t)\rangle$ is a nonincreasing function on $[0, \infty)$.

The proof of statement (ii) of Theorem 2.2 was inspired by some ideas of [12]. We feel that it may be helpful for the reader if we first give a heuristic outline. Let $u$ be as in Theorem 2.2(ii). Define

$$
\xi(t):=\langle|u(t)|, \psi(t)\rangle,
$$

where $t \geq s$. Lemma 5.2 states that the function $\xi(t)$ is nonincreasing. It has been proved in [29], where equations on smooth bounded domains $\Omega \subset \mathbb{R}^{N}$ are considered, that the corresponding function is in fact exponentially decreasing (uniformly for all $u$ ). It was also shown in [29], that this exponential 
decay can be used for the proof of an exponential separation estimate. In the case of $\Omega=\mathbb{R}^{N}, \xi$ may fail to be exponentially decreasing (uniformly in $u$ ), however, it is still useful in our analysis. To establish estimate (2.9) we use the following strategy. For a specified unit of time, $\tau_{0}$, we analyze the relative decrease of $u$ with respect to $\varphi$ during that time. More precisely, if $\left\|u\left(t+\tau_{0}\right)\right\|_{L^{\infty}\left(\mathbb{R}^{N}\right)} /\|u(t)\|_{L^{\infty}\left(\mathbb{R}^{N}\right)} \ll\left\|\varphi\left(t+\tau_{0}\right)\right\|_{L^{\infty}\left(\mathbb{R}^{N}\right)} /\|\varphi(t)\|_{L^{\infty}\left(\mathbb{R}^{N}\right)}$, then we simply move on from $t$ to $t+\tau_{0}$. If, on the other hand, $\| u(t+$ $\left.\tau_{0}\right)\left\|_{L^{\infty}\left(\mathbb{R}^{N}\right)} /\right\| u(t)\left\|_{L^{\infty}\left(\mathbb{R}^{N}\right)} \approx\right\| \varphi\left(t+\tau_{0}\right)\left\|_{L^{\infty}\left(\mathbb{R}^{N}\right)} /\right\| \varphi(t) \|_{L^{\infty}\left(\mathbb{R}^{N}\right)}$, that is, if $u$ does not drop "too much" compared to $\varphi$, then Lemma 5.4 below guarantees that $\xi\left(t+\tau_{0}\right) / \xi(t)<1-\epsilon$ for some fixed $\epsilon>0$ and Lemma 5.5 guarantees that in that case $\xi(t) \approx\|u(t)\|_{L^{\infty}\left(\mathbb{R}^{N}\right)} /\|\varphi(t)\|_{L^{\infty}\left(\mathbb{R}^{N}\right)}$. This "trade off" between the relative decay of $u$ with respect to $\varphi$ and the decay of $\xi$ is sufficient for the exponential separation estimate.

We start with some useful estimates on the functions $\varphi$ and $\psi$.

Lemma 5.3. (i) There is a constant $C_{1}$ depending only on the given quantities such that

$$
C_{1}^{-1} \leq\|\varphi(\cdot, t)\|_{L^{\infty}\left(\mathbb{R}^{N}\right)}\|\psi(\cdot, t)\|_{L^{\infty}\left(\mathbb{R}^{N}\right)} \leq C_{1} \quad(t \in \mathbb{R}) .
$$

(ii) For any $r>0$ there is a positive constant $C_{2}(r)$ depending only on $r$ and the given quantities such that

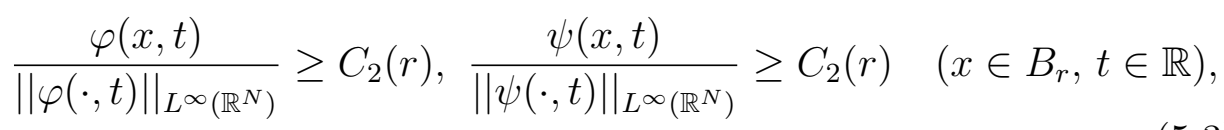

Proof. We first prove (ii). By Proposition 3.4 and (2.7), we have

$$
C_{\varphi}^{-1} \leq \frac{\|\varphi(\cdot, t)\|_{L^{\infty}\left(\mathbb{R}^{N}\right)}}{\|\varphi(\cdot, s)\|_{L^{\infty}\left(\mathbb{R}^{N}\right)}} \leq C_{\varphi} \quad(t, s \in[\tau, \tau+1], \tau \in \mathbb{R}),
$$

where $C_{\varphi}>0$ depends only on the given quantities. Referring to the Harnack inequality (Theorem 3.1), we see that estimate (5.3) for $\varphi$ is a consequence of (5.4), (2.6). The arguments for $\psi$ are similar.

Now, by Lemma $5.1,\langle\varphi(\cdot, t), \psi(\cdot, t)\rangle \equiv$ const. This in conjunction with (5.3), and (2.6) readily imply (5.2).

In the following text we denote by $f_{+}\left(f_{-}\right)$the positive (negative) part of a real valued function $f$. For the remainder of this section we define $C$ and $\tau_{0}$ by

$$
C=e^{d_{0}}, \quad c_{2} e^{\epsilon_{0} \tau_{0}}=4 C^{2},
$$


where $c_{2}$ and $\epsilon_{0}$ are as in Theorem 2.1. Note that $\tau_{0}$ depends only on the given quantities and that inequalities (2.7), (3.8) (applied to $\varphi$, with $s=0$, $t=1)$ force $\tau_{0}>2$.

Lemma 5.4. Let $u$ be a solution of (3.3) with $u_{0} \in L^{\infty}\left(\mathbb{R}^{N}\right)$ such that $\left\langle u_{0}, \psi(0)\right\rangle=0$ (so that $\langle u(t), \psi(t)\rangle=0$ for all $t \geq 0$ ). With $\tau_{0}$ and $C$ as in (5.5), suppose that $\left\|u\left(\tau_{0}\right)\right\|_{L^{\infty}\left(\mathbb{R}^{N}\right)}>C^{2}\left\|u_{0}\right\|_{L^{\infty}\left(\mathbb{R}^{N}\right)}$. Then there is a positive constant $\mu<1$ depending only on the given quantities such that if $\xi(t):=\langle|u(t)|, \psi(t)\rangle$ then

$$
\xi\left(\tau_{0}\right) \leq \mu \xi(0)
$$

Proof. Let $u_{1}, u_{2}$ be the positive solutions of (3.3) with initial conditions equal to $u_{0+}$ and $u_{0-}$, respectively. Note that by uniqueness of solutions of (3.3) we have $u=u_{1}-u_{2}$.

Our first goal is to prove the following claim. For all $r>1$ sufficiently large there exists $c_{10}(r)>0$, depending only on $r$ and the given quantities, such that

$$
c_{10}^{-1}(r) \leq \frac{u_{1}\left(x, \tau_{0}\right)}{u_{2}\left(x, \tau_{0}\right)} \leq c_{10}(r) \quad\left(x \in B_{r}\right) .
$$

First note that our assumptions and Proposition 3.4 imply that

$$
C\left\|u\left(\tau_{0}-1\right)\right\|_{L^{\infty}\left(\mathbb{R}^{N}\right)} \geq\left\|u\left(\tau_{0}\right)\right\|_{L^{\infty}\left(\mathbb{R}^{N}\right)}>C^{2}\left\|u_{0}\right\|_{L^{\infty}\left(\mathbb{R}^{N}\right)} \geq C\|u(1)\|_{L^{\infty}\left(\mathbb{R}^{N}\right)} .
$$

These inequalities and Corollary 3.6 imply the existence of $t_{0} \in\left(1, \tau_{0}-1\right]$ such that $\left\|u\left(t_{0}\right)\right\|_{L^{\infty}\left(\mathbb{R}^{N}\right)}=\left\|u\left(t_{0}\right)\right\|_{L^{\infty}\left(B_{\rho_{0}}\right)} \geq C\left\|u_{0}\right\|_{L^{\infty}\left(\mathbb{R}^{N}\right)}$. Without loss of generality we shall assume $\left\|u\left(t_{0}\right)\right\|_{L^{\infty}\left(\mathbb{R}^{N}\right)}=\left\|u_{+}\left(t_{0}\right)\right\|_{L^{\infty}\left(\mathbb{R}^{N}\right)}$ (otherwise replace $u$ by $-u$ ). These considerations and Proposition 3.4 imply the following estimates

$$
\begin{aligned}
C\left\|u_{0}\right\|_{L^{\infty}\left(\mathbb{R}^{N}\right)} & \leq\left\|u\left(t_{0}\right)\right\|_{L^{\infty}\left(B_{\rho_{0}}\right)}=\left\|u_{1}\left(t_{0}\right)-u_{2}\left(t_{0}\right)\right\|_{L^{\infty}\left(B_{\rho_{0}}\right)} \\
& <\left\|u_{1}\left(t_{0}\right)\right\|_{L^{\infty}\left(B_{\rho_{0}}\right)} \leq\left\|u_{1}\left(t_{0}\right)\right\|_{L^{\infty}\left(\mathbb{R}^{N}\right)} \\
& \leq \tilde{C}\left\|u_{0+}\right\|_{L^{\infty}\left(\mathbb{R}^{N}\right)} \leq \tilde{C}\left\|u_{0}\right\|_{L^{\infty}\left(\mathbb{R}^{N}\right)},
\end{aligned}
$$

where $\tilde{C}>0$ depends only on the given quantities. It then follows from Proposition 3.4 and the Harnack inequality (3.1) that

$$
C_{1}^{-1}(r)\left\|u_{0}\right\|_{L^{\infty}\left(\mathbb{R}^{N}\right)} \leq u_{1}\left(x, \tau_{0}\right) \leq C_{1}(r)\left\|u_{0}\right\|_{L^{\infty}\left(\mathbb{R}^{N}\right)} \quad\left(x \in B_{r}\right)
$$


for all $r \geq \rho_{0}$ and some $C_{1}(r)>0$ depending only on $r$ and the given quantities.

We next prove a similar estimate for $u_{2}$. The assumption $\left\langle u_{0}, \psi(0)\right\rangle=0$ and Lemma 5.1 imply

$$
\left\langle u_{1}(t), \psi(t)\right\rangle=\left\langle u_{2}(t), \psi(t)\right\rangle \equiv \text { const } \quad(t \geq 0) .
$$

Taking $r=\rho_{0}$ in (5.9), and combining the resulting inequality with (5.3), we obtain

$$
\begin{aligned}
\int_{\mathbb{R}^{N}} u_{2}(x, 1) \psi(x, 1) d x=\int_{\mathbb{R}^{N}} u_{1}(x, 1) \psi(x, 1) d x \\
=\int_{\mathbb{R}^{N}} u_{1}\left(x, \tau_{0}\right) \psi\left(x, \tau_{0}\right) d x \geq \tilde{C}_{3}\left\|u_{0}\right\|_{L^{\infty}\left(\mathbb{R}^{N}\right)}\left\|\psi\left(\cdot, \tau_{0}\right)\right\|_{L^{\infty}\left(\mathbb{R}^{N}\right)}
\end{aligned}
$$

where $\tilde{C}_{3}$ depends only on the given quantities. Now, by (5.2) and (5.4), $\left\|\psi\left(\cdot, \tau_{0}\right)\right\|_{L^{\infty}\left(\mathbb{R}^{N}\right)}$ can be estimated below in terms of $\|\psi(\cdot, 1)\|_{L^{\infty}\left(\mathbb{R}^{N}\right)}$, and hence

$$
\int_{\mathbb{R}^{N}} u_{2}(x, 1) \psi(x, 1) d x \geq C_{3}\left\|u_{0}\right\|_{L^{\infty}\left(\mathbb{R}^{N}\right)}\|\psi(\cdot, 1)\|_{L^{\infty}\left(\mathbb{R}^{N}\right)},
$$

with $C_{3}$ depending only on the given quantities. On the other hand, by Proposition 3.4, $\left\|u_{2}(1)\right\|_{L^{\infty}\left(\mathbb{R}^{N}\right)} \leq C\left\|u_{0}\right\|_{L^{\infty}\left(\mathbb{R}^{N}\right)}$ and we have the exponential estimate (2.6) for $\psi$. Therefore, for some $r_{0}=r_{0}\left(C_{3}, C, c_{1}\right)>0\left(c_{1}\right.$ is as in (2.6)) we must have

$$
\int_{B_{r_{0}}} u_{2}(x, 1) \psi(x, 1) d x \geq\left(C_{3} / 2\right)\left\|u_{0}\right\|_{L^{\infty}\left(\mathbb{R}^{N}\right)}\|\psi(\cdot, 1)\|_{L^{\infty}\left(\mathbb{R}^{N}\right)} .
$$

Consequently, there is a point $x_{0} \in B_{r_{0}}$ such that $u_{2}\left(x_{0}, 1\right) \geq C_{4}\left\|u_{0}\right\|_{L^{\infty}\left(\mathbb{R}^{N}\right)}$ with $C_{4}=C_{4}\left(r_{0}, C_{3}\right)$. This inequality in conjunction with (3.1) and Proposition 3.4 imply (5.9) with $u_{1}$ replaced by $u_{2}$. Obviously, inequality (5.9) used for both $u_{1}$ and $u_{2}$ implies (5.7), finishing the proof of the claim.

Fix $r=\rho_{0}$ in the inequalities (5.7) and (5.9) above. An elementary estimate using (5.7) gives

$$
\left|u_{1}\left(x, \tau_{0}\right)-u_{2}\left(x, \tau_{0}\right)\right| \leq\left(1-c_{10}^{-1}\left(\rho_{0}\right)\right)\left(u_{1}\left(x, \tau_{0}\right)+u_{2}\left(x, \tau_{0}\right)\right) \quad\left(x \in B_{\rho_{0}}\right) .
$$


Inequalities (5.9) (applied to both $u_{1}$ and $u_{2}$ ), (5.3), and (2.6) imply that for $i=1,2$

$$
\begin{aligned}
\int_{B_{\rho_{0}}} u_{i}\left(x, \tau_{0}\right) \psi\left(x, \tau_{0}\right) d x & \geq C_{5}\left\|u_{0}\right\|_{L^{\infty}\left(\mathbb{R}^{N}\right)}\left\|\psi\left(\cdot, \tau_{0}\right)\right\|_{L^{\infty}\left(\mathbb{R}^{N}\right)} \\
& \geq C_{6} \int_{\mathbb{R}^{N}} u_{i}\left(x, \tau_{0}\right) \psi\left(x, \tau_{0}\right) d x
\end{aligned}
$$

for some positive constants $C_{5}, C_{6}$ depending only on the given quantities. Using (5.11), (5.12), and Lemma 5.1 we obtain

$$
\begin{aligned}
\xi\left(\tau_{0}\right)= & \left\langle\left|u\left(\tau_{0}\right)\right|, \psi\left(\tau_{0}\right)\right\rangle=\left\langle\left|u_{1}\left(\tau_{0}\right)-u_{2}\left(\tau_{0}\right)\right|, \psi\left(\tau_{0}\right)\right\rangle \\
= & \int_{B_{\rho_{0}}}\left|u_{1}\left(\tau_{0}\right)-u_{2}\left(\tau_{0}\right)\right| \psi\left(\tau_{0}\right) d x+\int_{\mathbb{R}^{N} \backslash B_{\rho_{0}}}\left|u_{1}\left(\tau_{0}\right)-u_{2}\left(\tau_{0}\right)\right| \psi\left(\tau_{0}\right) d x \\
\leq & \left(1-c_{10}^{-1}\left(\rho_{0}\right)\right) \int_{B_{\rho_{0}}}\left(u_{1}\left(\tau_{0}\right)+u_{2}\left(\tau_{0}\right)\right) \psi\left(\tau_{0}\right) d x \\
& \quad \int_{\mathbb{R}^{N} \backslash B_{\rho_{0}}}\left(u_{1}\left(\tau_{0}\right)+u_{2}\left(\tau_{0}\right)\right) \psi\left(\tau_{0}\right) d x \\
= & \int_{\mathbb{R}^{N}}\left(u_{1}\left(\tau_{0}\right)+u_{2}\left(\tau_{0}\right)\right) \psi\left(\tau_{0}\right) d x-c_{10}^{-1}\left(\rho_{0}\right) \int_{B_{\rho_{0}}}\left(u_{1}\left(\tau_{0}\right)+u_{2}\left(\tau_{0}\right)\right) \psi\left(\tau_{0}\right) d x \\
\leq & \left(1-C_{6} c_{10}^{-1}\left(\rho_{0}\right)\right) \int_{\mathbb{R}^{N}}\left(u_{1}\left(\tau_{0}\right)+u_{2}\left(\tau_{0}\right)\right) \psi\left(\tau_{0}\right) d x \\
= & \left(1-C_{6} c_{10}^{-1}\left(\rho_{0}\right)\right) \int_{\mathbb{R}^{N}}\left(u_{1}(0)+u_{2}(0)\right) \psi(0) d x=\left(1-C_{6} c_{10}^{-1}\left(\rho_{0}\right)\right) \xi(0) .
\end{aligned}
$$

We thus get the desired claim with $\mu=1-C_{6} c_{10}^{-1}\left(\rho_{0}\right)$, which clearly depends only on the given quantities.

Lemma 5.5. Let $u$ be a solution of (3.3) with $u_{0} \in L^{\infty}\left(\mathbb{R}^{N}\right)$ such that $\left\langle u_{0}, \psi(0)\right\rangle=0$. Then there exists $\delta_{0}>0$ depending only on the given quantities such that the following implication holds (with $\xi(t)=\langle|u(t)|, \psi(t)\rangle$ ):

$$
\text { if } \xi(0) \leq \delta_{0} \frac{\left\|u_{0}\right\|_{L^{\infty}\left(\mathbb{R}^{N}\right)}}{\|\varphi(0)\|_{L^{\infty}\left(\mathbb{R}^{N}\right)}} \quad \text { then }\left\|u\left(\tau_{0}\right)\right\|_{L^{\infty}\left(\mathbb{R}^{N}\right)} \leq C^{2}\left\|u_{0}\right\|_{L^{\infty}\left(\mathbb{R}^{N}\right)} \text {. }
$$

Here $\tau_{0}$ and $C$ are the constants defined in (5.5). 
Proof. For $r>0$ to be specified below we write

$$
u_{0}(x)=\chi_{r}(x) u_{0}(x)+\left(1-\chi_{r}(x)\right) u_{0}(x)=u_{10}(x)+u_{20}(x),
$$

where $\chi_{r}: \mathbb{R}^{N} \rightarrow \mathbb{R}$ is a smooth function such that $0 \leq \chi_{r} \leq 1$ and $\chi_{r}(x)=1$ for $x \in B_{r}$ and $\chi_{r}(x)=0$ if $|x| \geq r+1$. Let $u_{i}, i=1,2$, be the solution of (3.3) with $u_{0}=u_{i 0}$ so that $u=u_{1}+u_{2}$. We first estimate $\left\|u_{2}\left(\tau_{0}\right)\right\|_{L^{\infty}\left(\mathbb{R}^{N}\right)}$. In that we can assume without loss of generality that $u_{2} \geq 0$. Indeed, otherwise, writing $u_{20}=u_{20+}-u_{20-}$, we would estimate each of the nonnegative solutions of $(3.3)$ with the initial condition $u_{20+}\left(u_{20-}\right)$. Define the set

$$
M=\left\{t \in\left(0, \tau_{0}\right]:\left\|u_{2}(t)\right\|_{L^{\infty}\left(\mathbb{R}^{N}\right)}=\left\|u_{2}(t)\right\|_{L^{\infty}\left(B_{\rho_{0}}\right)}\right\} .
$$

If $M$ is empty then by Corollary 3.6

$$
\left\|\left.u_{2}\left(\tau_{0}\right)\right|_{L^{\infty}\left(\mathbb{R}^{N}\right)} \leq\right\| u_{20}\left\|_{L^{\infty}\left(\mathbb{R}^{N}\right)} \leq\right\| u_{0} \|_{L^{\infty}\left(\mathbb{R}^{N}\right)} .
$$

If, on the other hand, $M$ is nonempty, then by Proposition 3.4 and Lemma 3.3 we have the following estimates for any $t_{0} \in M$

$$
\begin{aligned}
\left\|u_{2}\left(\tau_{0}\right)\right\|_{L^{\infty}\left(\mathbb{R}^{N}\right)} & \leq C^{\tau_{0}-t_{0}}\left\|u_{2}\left(t_{0}\right)\right\|_{L^{\infty}\left(\mathbb{R}^{N}\right)}=C^{\tau_{0}-t_{0}}\left\|u_{2}\left(t_{0}\right)\right\|_{L^{\infty}\left(B_{\rho_{0}}\right)} \\
& \leq C^{\tau_{0}} c(N)\left(t_{0}\right)^{N / 2} /\left(r-\rho_{0}\right)^{N}\left\|u_{0}\right\|_{L^{\infty}\left(\mathbb{R}^{N}\right)} .
\end{aligned}
$$

Choosing $r=r_{0}>1$ sufficiently large (independently of $u_{0}$ ) we obtain $\left\|u_{2}\left(\tau_{0}\right)\right\|_{L^{\infty}\left(\mathbb{R}^{N}\right)} \leq\left\|u_{0}\right\|_{L^{\infty}\left(\mathbb{R}^{N}\right)}$ in this case as well.

Let us now estimate $u_{1}\left(\tau_{0}\right)$. By (3.4) we have for any $x \in \mathbb{R}^{N}$

$$
\begin{aligned}
\left|u_{1}\left(x, \tau_{0}\right)\right| & \leq \int_{\mathbb{R}^{N}} \Gamma\left(x, \tau_{0} ; y, 0\right)\left|u_{10}(y)\right| d y \\
& \leq \int_{B_{r_{0}+1}} \frac{e^{d_{0} \tau_{0}}}{\left(4 \pi \tau_{0}\right)^{N / 2}} e^{\frac{-|x-y|^{2}}{4 \tau_{0}}}\left|u_{0}(y)\right| d y .
\end{aligned}
$$

It is clear to see that there exists $r_{1}>1$ depending only on $N, d_{0}, \tau_{0}, r_{0}$, and $C$ such that if $x \in \mathbb{R}^{N} \backslash B_{r_{1}}$ then (5.16) implies $\left|u_{1}\left(x, \tau_{0}\right)\right| \leq\left(C^{2}-1\right)\left\|u_{0}\right\|_{L^{\infty}\left(\mathbb{R}^{N}\right)}$.

We finally need to estimate $\left|u_{1}\left(x, \tau_{0}\right)\right|$ for $x \in B_{r_{1}}$. For that we let $C_{2}=$ $C_{2}(r)$ be as in (5.3) with $r=r_{0}+1$. Using successively (5.16), (5.3), (5.2), 
and the assumption on $\xi(0)$, we obtain for any $x \in B_{r_{1}}$

$$
\begin{aligned}
\left|u_{1}\left(x, \tau_{0}\right)\right| & \leq C_{3} \int_{B_{r_{0}+1}}\left|u_{0}(y)\right| d y \leq C_{3} C_{2}^{-1} \int_{B_{r_{0}+1}}\left|u_{0}(y)\right| \frac{\psi(y, 0)}{\|\psi(\cdot, 0)\|_{L^{\infty}\left(\mathbb{R}^{N}\right)}} d y \\
& \leq C_{3} C_{2}^{-1} C_{1}\|\varphi(\cdot, 0)\|_{L^{\infty}\left(\mathbb{R}^{N}\right)} \int_{\mathbb{R}^{N}}\left|u_{0}(y)\right| \psi(y, 0) d y \\
& =C_{3} C_{2}^{-1} C_{1}\|\varphi(\cdot, 0)\|_{L^{\infty}\left(\mathbb{R}^{N}\right)} \xi(0) \\
& \leq C_{3} C_{2}^{-1} C_{1} \delta_{0}\left\|u_{0}\right\|_{L^{\infty}\left(\mathbb{R}^{N}\right)}
\end{aligned}
$$

where $C_{3}>0$ is independent of $u_{0}$. Choosing $\delta_{0}=C_{2}\left(C^{2}-1\right) /\left(C_{3} C_{1}\right)$ we obtain $\left|u_{1}\left(x, \tau_{0}\right)\right| \leq\left(C^{2}-1\right)\left\|u_{0}\right\|_{L^{\infty}\left(\mathbb{R}^{N}\right)}$. Combining the above results we have proved that with $\delta_{0}$ chosen as above we have

$$
\left\|\left.u\left(\tau_{0}\right)\right|_{L^{\infty}\left(\mathbb{R}^{N}\right)} \leq\right\| u_{1}\left(\tau_{0}\right)\left\|_{L^{\infty}\left(\mathbb{R}^{N}\right)}+\right\| u_{2}\left(\tau_{0}\right)\left\|_{L^{\infty}\left(\mathbb{R}^{N}\right)} \leq C^{2}\right\| u_{0} \|_{L^{\infty}\left(\mathbb{R}^{N}\right)},
$$

finishing the proof of Lemma 5.5.

With the above preparation we can complete the proof of Theorem 2.2 using similar arguments as in [12, Proof of Theorem 4.2].

Proof of Theorem 2.2(ii). The symbols $\tau_{0}, C$ have the same meanings as above, see the paragraph preceding Lemma 5.4, and $\delta_{0}$ is as in Lemma 5.5.

Let $t \geq s$ be arbitrary and let $u(t)=u\left(\cdot, t, s, u_{0}\right)$ with $u_{0} \in X_{2}(s)$. Define

$$
t_{1}=\inf \left\{\tau \in[s, t]: \xi(r)<\delta_{0} \frac{\|u(r)\|_{L^{\infty}\left(\mathbb{R}^{N}\right)}}{\|\varphi(r)\|_{L^{\infty}\left(\mathbb{R}^{N}\right)}} \text { for all } r \in[\tau, t]\right\}
$$

or set $t_{1}=t$ if this set is empty. Note that the definition of $t_{1}$ implies that we either have

$$
\xi\left(t_{1}\right) \geq \delta_{0} \frac{\left\|u\left(t_{1}\right)\right\|_{L^{\infty}\left(\mathbb{R}^{N}\right)}}{\left\|\varphi\left(t_{1}\right)\right\|_{L^{\infty}\left(\mathbb{R}^{N}\right)}}
$$

or $\xi\left(t_{1}\right)<\delta_{0}\left\|u\left(t_{1}\right)\right\|_{L^{\infty}\left(\mathbb{R}^{N}\right)} /\left\|\varphi\left(t_{1}\right)\right\|_{L^{\infty}\left(\mathbb{R}^{N}\right)}$ and then $t_{1}=s$. Also, using (2.6) and (5.2), we get

$$
\xi(s)=\langle|u(s)|, \psi(s)\rangle \leq C_{2}\|u(s)\|_{L^{\infty}\left(\mathbb{R}^{N}\right)}\|\psi(s)\|_{L^{\infty}\left(\mathbb{R}^{N}\right)} \leq C_{1} C_{2} \frac{\|u(s)\|_{L^{\infty}\left(\mathbb{R}^{N}\right)}}{\|\varphi(s)\|_{L^{\infty}\left(\mathbb{R}^{N}\right)}}
$$

We next prove the estimate

$$
\frac{\left\|u\left(t_{1}\right)\right\|_{L^{\infty}\left(\mathbb{R}^{N}\right)}}{\left\|\varphi\left(t_{1}\right)\right\|_{L^{\infty}\left(\mathbb{R}^{N}\right)}} \leq \tilde{C} \tilde{\nu}^{\left(t_{1}-s\right)} \frac{\|u(s)\|_{L^{\infty}\left(\mathbb{R}^{N}\right)}}{\|\varphi(s)\|_{L^{\infty}\left(\mathbb{R}^{N}\right)}},
$$


where $\tilde{C}>0, \tilde{\nu} \in(0,1)$ depend only on the given quantities. If $t_{1}-s<\tau_{0}$, then such an estimate readily follows from Proposition 3.4 and (2.7); in fact

$$
\frac{\left\|u\left(t_{1}\right)\right\|_{L^{\infty}\left(\mathbb{R}^{N}\right)}}{\left\|\varphi\left(t_{1}\right)\right\|_{L^{\infty}\left(\mathbb{R}^{N}\right)}} \leq C_{3} \frac{\|u(s)\|_{L^{\infty}\left(\mathbb{R}^{N}\right)}}{\|\varphi(s)\|_{L^{\infty}\left(\mathbb{R}^{N}\right)}}
$$

for some $C_{3}>0$ depending only on the given quantities.

Next assume $t_{1}-s \geq \tau_{0}$. Set $\kappa:=\left[\left(t_{1}-s\right) / \tau_{0}\right]$ (the integer part of $\left.\left(t_{1}-s\right) / \tau_{0}\right)$ and $\tilde{\tau}_{j}=t_{1}-\kappa \tau_{0}+j \tau_{0}, j=0, \ldots, \kappa$. Lemma 5.4 implies that for each $j=0, \ldots, \kappa-1$ we have the following two (mutually exclusive) possibilities:

(a) $\left\|u\left(\tilde{\tau}_{j+1}\right)\right\|_{L^{\infty}\left(\mathbb{R}^{N}\right)} \leq C^{2}\left\|u\left(\tilde{\tau}_{j}\right)\right\|_{L^{\infty}\left(\mathbb{R}^{N}\right)}$,

(b) $\left\|u\left(\tilde{\tau}_{j+1}\right)\right\|_{L^{\infty}\left(\mathbb{R}^{N}\right)}>C^{2}\left\|u\left(\tilde{\tau}_{j}\right)\right\|_{L^{\infty}\left(\mathbb{R}^{N}\right)}$ and then $\xi\left(\tilde{\tau}_{j+1}\right) \leq \mu \xi\left(\tilde{\tau}_{j}\right)$.

Suppose that (a) occurs exactly $l$ times (hence (b) occurs $\kappa-l$ times). We distinguish two scenarios:

(j) $l \geq \kappa\left(1-\ln 2 /\left(\left(\tau_{0}-2\right) \ln C\right)\right)$,

(jj) $l<\kappa\left(1-\ln 2 /\left(\left(\tau_{0}-2\right) \ln C\right)\right)$.

Assume that (j) holds. Note that by the definition of $C$ and $\tau_{0}$ (cf. (5.5)), and by Proposition 3.4 and (2.7) we have

$$
\left\|u\left(\tilde{\tau}_{j+1}\right)\right\|_{L^{\infty}\left(\mathbb{R}^{N}\right)} \leq C^{\tau_{0}}\left\|u\left(\tilde{\tau}_{j}\right)\right\|_{L^{\infty}\left(\mathbb{R}^{N}\right)},
$$

and

$$
\left\|\varphi\left(\tilde{\tau}_{j+1}\right)\right\|_{L^{\infty}\left(\mathbb{R}^{N}\right)} \geq 4 C^{2}\left\|\varphi\left(\tilde{\tau}_{j}\right)\right\|_{L^{\infty}\left(\mathbb{R}^{N}\right)} .
$$

Using (5.23), (5.22) $\kappa-l$ times and (a) $l$ times we obtain

$$
\begin{aligned}
\frac{\left\|u\left(t_{1}\right)\right\|_{L^{\infty}\left(\mathbb{R}^{N}\right)}}{\left\|\varphi\left(t_{1}\right)\right\|_{L^{\infty}\left(\mathbb{R}^{N}\right)}} & \leq 4^{-\kappa} C^{\tau_{0}(\kappa-\ell)-2(\kappa-l)} \frac{\left\|u\left(\tilde{\tau}_{0}\right)\right\|_{L^{\infty}\left(\mathbb{R}^{N}\right)}}{\left\|\varphi\left(\tilde{\tau}_{0}\right)\right\|_{L^{\infty}\left(\mathbb{R}^{N}\right)}} \\
& \leq C_{3} \frac{1}{2^{\kappa}} \frac{\|u(s)\|_{L^{\infty}\left(\mathbb{R}^{N}\right)}}{\|\varphi(s)\|_{L^{\infty}\left(\mathbb{R}^{N}\right)}}
\end{aligned}
$$

(in the last estimate we used (5.21) with $t_{1}$ replaced by $\tilde{\tau}_{0}$ and $(\mathrm{j})$ ). 
We now show that a similar estimate holds in the case (jj) as well. This time we use (5.18), the nonincrease of $\xi$ (see Lemma 5.2), estimate on $\xi$ in (b) $(\kappa-l$ times $)$, and (5.19), to derive

$$
\begin{aligned}
\frac{\left\|u\left(t_{1}\right)\right\|_{L^{\infty}\left(\mathbb{R}^{N}\right)}}{\left\|\varphi\left(t_{1}\right)\right\|_{L^{\infty}\left(\mathbb{R}^{N}\right)}} & \leq \delta_{0}^{-1} \xi\left(t_{1}\right) \leq \delta_{0}^{-1} \mu^{\kappa-l} \xi\left(t_{1}-\kappa \tau_{0}\right) \\
& \leq \delta_{0}^{-1}\left(\mu^{\ln 2 /\left(\left(\tau_{0}-2\right) \ln C\right)}\right)^{\kappa} \xi(s) \\
& \leq \delta_{0}^{-1} C_{1} C_{2} \tilde{\mu}^{\kappa} \frac{\|u(s)\|_{L^{\infty}\left(\mathbb{R}^{N}\right)}}{\|\varphi(s)\|_{L^{\infty}\left(\mathbb{R}^{N}\right)}}
\end{aligned}
$$

where $\tilde{\mu}=\mu^{\ln 2 /\left(\left(\tau_{0}-2\right) \ln C\right)}<1$. Since $\kappa+1>\left(t_{1}-s\right) / \tau_{0}$, estimates (5.24), (5.25) give (5.20) with $\tilde{\nu}=\max \left\{2^{-1 / \tau_{0}}, \tilde{\mu}^{1 / \tau_{0}}\right\}$.

To conclude the proof, we now estimate $\|u(\tilde{t})\|_{L^{\infty}\left(\mathbb{R}^{N}\right)} /\|\varphi(\tilde{t})\|_{L^{\infty}\left(\mathbb{R}^{N}\right)}$ with $\tilde{t}=t$ in terms of its value at $\tilde{t}=t_{1}$. The definition of $t_{1}$ in (5.17) and Lemma 5.5 imply that if we set $\tilde{\tau}_{j}=t_{1}+j \tau_{0}, j=0, \ldots,\left[\left(t-t_{1}\right) / \tau_{0}\right]$, we necessarily have $\left\|u\left(\tilde{\tau}_{j+1}\right)\right\|_{L^{\infty}\left(\mathbb{R}^{N}\right)} \leq C^{2}\left\|u\left(\tilde{\tau}_{j}\right)\right\|_{L^{\infty}\left(\mathbb{R}^{N}\right)}$ for each such $j$. This situation falls into the scenario $(\mathrm{j})$ considered above and hence

$$
\frac{\|u(t)\|_{L^{\infty}\left(\mathbb{R}^{N}\right)}}{\|\varphi(t)\|_{L^{\infty}\left(\mathbb{R}^{N}\right)}} \leq C_{3}\left(\frac{1}{2}\right)^{\left[\left(t-t_{1}\right) / \tau_{0}\right]} \frac{\left\|u\left(t_{1}\right)\right\|_{L^{\infty}\left(\mathbb{R}^{N}\right)}}{\left\|\varphi\left(t_{1}\right)\right\|_{L^{\infty}\left(\mathbb{R}^{N}\right)}}
$$

Combining estimates (5.20) and (5.26), we conclude that for some constants $\tilde{C}_{1}>0, \nu \in(0,1)$ depending only on the given quantities we have

$$
\frac{\|u(t)\|_{L^{\infty}\left(\mathbb{R}^{N}\right)}}{\|\varphi(t)\|_{L^{\infty}\left(\mathbb{R}^{N}\right)}} \leq \tilde{C}_{1} \nu^{(t-s)} \frac{\|u(s)\|_{L^{\infty}\left(\mathbb{R}^{N}\right)}}{\|\varphi(s)\|_{L^{\infty}\left(\mathbb{R}^{N}\right)}} .
$$

Taking $\gamma=-\ln \nu$, we obtain the desired inequality. This finishes the proof of statement (ii).

To conclude this section, we prove that the exponential separation estimate holds with the $L^{\infty}$-norms replaced by $L^{2}$-norms, as stated in Remark 2.3. We use the following result.

Lemma 5.6. Given any $\nu>0$ there exists a constant $K_{0}=K_{0}\left(\rho_{0}, \nu\right)$ with the following property. Let $u$ be a solution of (1.1) on an interval $\left(t_{1}, t_{2}\right]$ having the initial value $u\left(t_{1}\right) \in L^{2}\left(\mathbb{R}^{N}\right) \cap L^{\infty}\left(\mathbb{R}^{N}\right)$ and satisfying the estimate

$$
\|u(t)\|_{L^{2}\left(\mathbb{R}^{N}\right)} \geq K_{0}\|u(t)\|_{L^{\infty}\left(\mathbb{R}^{N}\right)} \quad\left(t \in\left(t_{1}, t_{2}\right]\right) .
$$

Then

$$
\|u(t)\|_{L^{2}\left(\mathbb{R}^{N}\right)} \leq e^{\nu\left(t-t_{1}\right)}\left\|u\left(t_{1}\right)\right\|_{L^{2}\left(\mathbb{R}^{N}\right)} \quad\left(t \in\left[t_{1}, t_{2}\right]\right) .
$$


Proof. Let $K_{1}:=\left|B_{\rho_{0}}\right|^{1 / 2}$, so that for any $t \in\left(t_{1}, t_{2}\right]$

$$
\|u(t)\|_{L^{2}\left(B_{\rho_{0}}\right)} \leq K_{1}\|u(t)\|_{L^{\infty}\left(B_{\rho_{0}}\right)} .
$$

We claim that the conclusion of the lemma holds with $K_{0}=K_{0}\left(\rho_{0}, \nu\right)$ chosen so large that

$$
-2 \nu+2\left(d_{0}-\nu\right) \frac{K_{1}^{2}}{K_{0}^{2}-K_{1}^{2}}<0
$$

$\left(d_{0}\right.$ is as in hypothesis $\left.(\mathrm{A})\right)$. To show this assume that (5.27) is satisfied. A standard energy inequality for (1.1) and (A) give

$$
\begin{aligned}
\frac{d}{d t}\left(e^{-2 \nu t}\|u(t)\|_{L^{2}\left(\mathbb{R}^{N}\right)}^{2}\right) & \leq 2 \int_{\mathbb{R}^{N}}(a(x, t)-\nu) u^{2}(x, t) d x \\
& \leq-2 \nu \int_{\mathbb{R}^{N} \backslash B_{\rho_{0}}} u^{2}(x, t) d x+2\left(d_{0}-\nu\right) \int_{B_{\rho_{0}}} u^{2}(x, t) d x
\end{aligned}
$$

Next, by (5.27) we have

$$
\begin{aligned}
\int_{\mathbb{R}^{N} \backslash B_{\rho_{0}}} u^{2}(x, t) d x & +\int_{B_{\rho_{0}}} u^{2}(x, t) d x \\
& =\|u(t)\|_{L^{2}\left(\mathbb{R}^{N}\right)}^{2} \geq K_{0}^{2}\|u(t)\|_{L^{\infty}\left(\mathbb{R}^{N}\right)}^{2} \geq \frac{K_{0}^{2}}{K_{1}^{2}}\|u(t)\|_{L^{2}\left(B_{\rho_{0}}\right)}^{2},
\end{aligned}
$$

and hence

$$
\|u(t)\|_{L^{2}\left(B_{\rho_{0}}\right)}^{2} \leq \frac{K_{1}^{2}}{K_{0}^{2}-K_{1}^{2}}\|u(t)\|_{L^{2}\left(\mathbb{R}^{N} \backslash B_{\rho_{0}}\right)}^{2} .
$$

Substituting this in (5.30) and using (5.29), we discover that $e^{-\nu t}\|u(t)\|_{L^{2}\left(\mathbb{R}^{N}\right)}$ is nonincreasing in $t$, which gives (5.28).

Proof of Remark 2.3. In this proof $C_{7}-C_{10}$ denote positive constants depending only on the given quantities (appearing in hypotheses (A) and (H1)).

By the Gaussian estimate on the Green's function, see (3.5), we have the following estimates on the solutions

$$
\begin{aligned}
& \left\|u\left(\cdot, s+1 ; s, u_{0}\right)\right\|_{L^{\infty}\left(\mathbb{R}^{N}\right)} \leq C_{7}\left\|u_{0}\right\|_{L^{2}\left(\mathbb{R}^{N}\right)} \quad(s \in \mathbb{R}), \\
& \left\|u\left(\cdot, t ; s, u_{0}\right)\right\|_{L^{2}\left(\mathbb{R}^{N}\right)} \leq C_{7}\left\|u_{0}\right\|_{L^{2}\left(\mathbb{R}^{N}\right)} \quad(s+1 \geq t \geq s)
\end{aligned}
$$

whenever $u_{0} \in L^{2}\left(\mathbb{R}^{N}\right)$. The estimates on $\varphi$ given in Theorem 2.1 imply

$$
C_{8}^{-1} \leq \frac{\|\varphi(\cdot, t)\|_{L^{2}\left(\mathbb{R}^{N}\right)}}{\|\varphi(\cdot, t)\|_{L^{\infty}\left(\mathbb{R}^{N}\right)}} \leq C_{8} \quad(t \in \mathbb{R})
$$


(the lower bound follows from (2.7) and (5.31)).

Estimates (5.32) and (5.33) readily imply that if $t \in[s, s+1]$, the $L^{\infty}$ norms in $(2.9)$ can be replaced by $L^{2}$-norms, adjusting the constant $C$ if necessary. It is therefore sufficient to consider the case $t>s+1$.

By (5.33) and (2.7),

$$
\frac{\|\varphi(\cdot, t)\|_{L^{2}\left(\mathbb{R}^{N}\right)}}{\|\varphi(\cdot, s)\|_{L^{2}\left(\mathbb{R}^{N}\right)}} \geq C_{9} e^{\epsilon_{0}(t-s)}
$$

Now, (2.9) (with $s$ replaced by $s+1$ and $u_{0}$ replaced by $u\left(\cdot, s+1 ; s, u_{0}\right)$ ) in conjunction with (5.31), (5.33) and (5.34), give

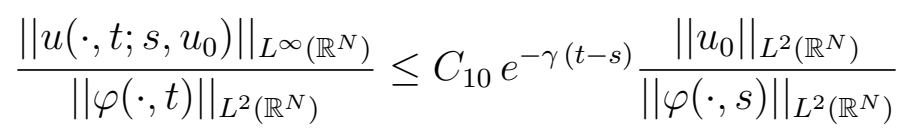

for any $t>s+1$ and $u_{0} \in X^{2}(s) \cap L^{2}\left(\mathbb{R}^{N}\right)$. There is one last $L^{\infty}$-norm in (5.35) to be replaced by the $L^{2}$-norm. Let $K_{0}$ be as in Lemma 5.6 with $\nu=\epsilon_{0} / 2$. Denoting $u(t)=u\left(\cdot, t ; s, u_{0}\right)$ for brevity, set

$$
t_{1}:=\inf \left\{\sigma \in[s+1, t]:\|u(\tau)\|_{L^{2}\left(\mathbb{R}^{N}\right)}>K_{0}\|u(\tau)\|_{L^{\infty}\left(\mathbb{R}^{N}\right)} \text { for all } \tau \in[\sigma, t]\right\}
$$

if the set is nonempty; otherwise set $t_{1}:=t$. If $t_{1}>s+1$ then $\left\|u\left(t_{1}\right)\right\|_{L^{2}\left(\mathbb{R}^{N}\right)} \leq$ $K_{0}|| u\left(t_{1}\right) \|_{L^{\infty}\left(\mathbb{R}^{N}\right)}$, hence by $(5.35)$,

$$
\frac{\left\|u\left(t_{1}\right)\right\|_{L^{2}\left(\mathbb{R}^{N}\right)}}{\left\|\varphi\left(t_{1}\right)\right\|_{L^{2}\left(\mathbb{R}^{N}\right)}} \leq K_{0} C_{10} e^{-\gamma\left(t_{1}-s\right)} \frac{\left\|u_{0}\right\|_{L^{2}\left(\mathbb{R}^{N}\right)}}{\|\varphi(\cdot, s)\|_{L^{2}\left(\mathbb{R}^{N}\right)}} .
$$

If $t_{1}=t$ we just use this estimate. If $t_{1} \in[s+1, t)$, we first combine (5.34) with the estimate $\|u(t)\|_{L^{2}\left(\mathbb{R}^{N}\right)} \leq e^{\epsilon_{0}\left(t-t_{1}\right) / 2}\left\|u\left(t_{1}\right)\right\|_{L^{2}\left(\mathbb{R}^{N}\right)}$ (which follows from Lemma 5.6 and the choice $\left.\nu=\epsilon_{0} / 2\right)$, to obtain

$$
\frac{\|u(t)\|_{L^{2}\left(\mathbb{R}^{N}\right)}}{\|\varphi(t)\|_{L^{2}\left(\mathbb{R}^{N}\right)}} \leq C_{9}^{-1} e^{-\frac{\epsilon_{0}}{2}\left(t-t_{1}\right)} \frac{\left\|u\left(t_{1}\right)\right\|_{L^{2}\left(\mathbb{R}^{N}\right)}}{\left\|\varphi\left(t_{1}\right)\right\|_{L^{2}\left(\mathbb{R}^{N}\right)}} .
$$

Subsequently we estimate the right hand side of (5.37) using (5.36). In either, case we get the desired estimate $(2.10)$ with a suitable constant $\tilde{C}$ and with $\tilde{\gamma}=\min \left\{\gamma, \epsilon_{0} / 2\right\}$. 


\section{Proof of Theorem 2.4}

In the whole section we assume that (A) holds and that for some $s_{0} \in \mathbb{R}$ there exists a solution $\phi$ of (1.1) satisfying (2.3). The constants $\tilde{C}_{0}, \tilde{\epsilon}_{0}$ are as in (2.3). We first show the following estimates on $\phi$ which are analogous to estimates on the function $\phi_{R}$ proved above.

Lemma 6.1. The following statements hold true.

(i) There is a constant $c_{1}$ depending only on the given quantities such that

$$
\frac{|\phi(x, t)|}{\|\phi(\cdot, t)\|_{L^{\infty}\left(\mathbb{R}^{N}\right)}} \leq c_{1}\left(e^{-\tilde{\epsilon}_{0}\left(t-s_{0}\right)}+e^{-\sqrt{\epsilon_{0}}|x|}\right) \quad\left(x \in \mathbb{R}^{N}, t \geq s_{0}\right) .
$$

(ii) Given any $\tau>0$ there is a constant $c_{2}(\tau)>0$ depending only on $\tau$ and the given quantities such that

$$
\left(c_{2}(\tau)\right)^{-1} \leq \frac{\|\phi(\cdot, t)\|_{L^{\infty}\left(\mathbb{R}^{N}\right)}}{\|\phi(\cdot, s)\|_{L^{\infty}\left(\mathbb{R}^{N}\right)}} \leq c_{2}(\tau) \quad\left(t, s \geq s_{0},|t-s| \leq \tau\right) .
$$

Proof. The proof of statement (i) is a straightforward modification of the proof of Lemma 4.4 and is omitted. Statement (ii) follows readily from (2.3) and Proposition 3.4.

The proof of Theorem 2.4 is based on a series of lemmas below. The first one states that if the positive part of $\phi(\cdot, \tau)$ significantly dominates the negative part of $\phi(\cdot, \tau)$ then $\phi(\cdot, t)$ becomes (and stays) positive in $B_{\rho_{0}}$ for all $t \geq \tau+4$.

Lemma 6.2. There exist $T_{0}>0, \alpha>1, c_{3}>0$ depending only on the given quantities with the following property. If for some $\tau \geq s_{0}+T_{0}$ one has

$$
\left\|\phi^{+}(\cdot, \tau)\right\|_{L^{\infty}\left(\mathbb{R}^{N}\right)} \geq \alpha\left\|\phi^{-}(\cdot, \tau)\right\|_{L^{\infty}\left(\mathbb{R}^{N}\right)}
$$

then

$$
\phi(x, t) \geq c_{3}\|\phi(\cdot, t)\|_{L^{\infty}\left(\mathbb{R}^{N}\right)} \quad\left(x \in B_{\rho_{0}}, t \geq \tau+4\right) .
$$

Proof. Let us first fix $T_{0}>3$ such that

$$
c_{1} e^{-\tilde{\epsilon}_{0} T_{0}} \leq 1 / 4, \quad \tilde{C}_{0} e^{\tilde{\epsilon}_{0} T_{0}-3 d_{0}}>1,
$$


where $c_{1}$ is as in (6.1). Fix further $R \geq \rho_{0}+1$ such that $c_{1} e^{-\sqrt{\tilde{\epsilon}_{0}}(R-1)} \leq 1 / 4$. Obviously, $T_{0}$ and $R$ depend only on the given quantities and (6.1) implies that

$$
|\phi(x, t)| \leq(1 / 2)\|\phi(\cdot, t)\|_{L^{\infty}\left(\mathbb{R}^{N}\right)} \quad\left(|x| \geq R-1, t \geq s_{0}+T_{0}\right) .
$$

We are going to use Theorem 3.2 with $R$ as above, $\tau$ as in (6.3), $\tau_{i}=\tau+i$, $i=1,2,3$, and $\tau_{4}=\tau+3+T_{0}$. We get

$$
\begin{gathered}
\phi(x, t) \geq \kappa\left\|\phi^{+}\right\|_{L^{\infty}\left(B_{R} \times(\tau+1, \tau+2)\right)}-\left\|e^{d_{0}\left(3+T_{0}\right)} \phi^{-}\right\|_{L^{\infty}\left(B_{R+1} \times\left(\tau, \tau+3+T_{0}\right)\right)} \\
\left(|x| \leq R, t \in\left[\tau+3, \tau+3+T_{0}\right)\right),
\end{gathered}
$$

where $\kappa>0$ depends only on the given quantities and without loss of generality we can assume $\kappa \in(0,1]$. By a comparison argument, $u\left(x, t ; \tau, \phi^{-}(\cdot, \tau)\right) \geq$ $\phi^{-}(x, t), x \in \mathbb{R}^{N}, t \geq \tau$. Then using the maximum principle we get

$$
\begin{aligned}
\left\|\phi^{-}\right\|_{L^{\infty}\left(B_{R+1} \times\left(\tau, \tau+3+T_{0}\right)\right)} & \leq \sup _{t \in\left[\tau, \tau+3+T_{0}\right]}\left\|\phi^{-}(\cdot, t)\right\|_{L^{\infty}\left(\mathbb{R}^{N}\right)} \\
& \leq \sup _{t \in\left[\tau, \tau+3+T_{0}\right]}\left\|u\left(\cdot, t ; \tau, \phi^{-}(\cdot, \tau)\right)\right\|_{L^{\infty}\left(\mathbb{R}^{N}\right)} \\
& \leq e^{d_{0}\left(3+T_{0}\right)}\left\|\phi^{-}(\cdot, \tau)\right\|_{L^{\infty}\left(\mathbb{R}^{N}\right)}
\end{aligned}
$$

As a consequence of (6.7) and (6.8),

$$
\begin{gathered}
\phi(x, t) \geq \kappa\left\|\phi^{+}\right\|_{L^{\infty}\left(B_{R} \times(\tau+1, \tau+2)\right)}-e^{2 d_{0}\left(3+T_{0}\right)}\left\|\phi^{-}(\cdot, \tau)\right\|_{L^{\infty}\left(\mathbb{R}^{N}\right)} \\
\left(|x| \leq R, t \in\left[\tau+3, \tau+3+T_{0}\right)\right) .
\end{gathered}
$$

We claim that the assertion of the lemma holds with

$$
\alpha=\frac{e^{2 d_{0}\left(3+T_{0}\right)}}{\kappa \tilde{C}_{0}},
$$

(note that $\alpha>1$ as $\kappa \leq 1$ and $\tilde{C}_{0} \leq 1$ ). Using assumptions (2.3), (6.3) and Proposition 3.4, we obtain for any $s \in\left[\tau, \tau+3+T_{0}\right]$

$$
\begin{aligned}
\|\phi(\cdot, s)\|_{L^{\infty}\left(\mathbb{R}^{N}\right)} & \geq \tilde{C}_{0} e^{\tilde{\epsilon}_{0}(s-\tau)}\|\phi(\cdot, \tau)\|_{L^{\infty}\left(\mathbb{R}^{N}\right)}=\tilde{C}_{0} e^{\tilde{\epsilon}_{0}(s-\tau)}\left\|\phi^{+}(\cdot, \tau)\right\|_{L^{\infty}\left(\mathbb{R}^{N}\right)} \\
& \geq \tilde{C}_{0} e^{\tilde{\epsilon}_{0}(s-\tau)} \alpha\left\|\phi^{-}(\cdot, \tau)\right\|_{L^{\infty}\left(\mathbb{R}^{N}\right)} \\
& \geq \tilde{C}_{0} e^{\tilde{\epsilon}_{0}(s-\tau)} \alpha e^{-d_{0}(s-\tau)}\left\|\phi^{-}(\cdot, s)\right\|_{L^{\infty}\left(\mathbb{R}^{N}\right)}>\left\|\phi^{-}(\cdot, s)\right\|_{L^{\infty}\left(\mathbb{R}^{N}\right)},
\end{aligned}
$$


where we used the definition of $\alpha$ in the last inequality. Using (6.11), (6.6) and (2.3), we obtain

$$
\begin{aligned}
\left\|\phi^{+}(\cdot, s)\right\|_{L^{\infty}\left(B_{R}\right)} & =\left\|\phi^{+}(\cdot, s)\right\|_{L^{\infty}\left(\mathbb{R}^{N}\right)}=\|\phi(\cdot, s)\|_{L^{\infty}\left(\mathbb{R}^{N}\right)} \\
& \geq \tilde{C}_{0} e^{\tilde{\epsilon}_{0}(s-\tau)}\|\phi(\cdot, \tau)\|_{L^{\infty}\left(\mathbb{R}^{N}\right)} \quad\left(s \in\left[\tau, \tau+3+T_{0}\right]\right) .
\end{aligned}
$$

Plugging (6.12) with $s=\tau+1$ into (6.9) and using (6.3), we obtain

$$
\begin{gathered}
\phi(x, t) \geq \kappa \tilde{C}_{0} e^{\tilde{\epsilon}_{0}}\|\phi(\cdot, \tau)\|_{L^{\infty}\left(\mathbb{R}^{N}\right)}-\left(e^{2 d_{0}\left(3+T_{0}\right)} / \alpha\right)\|\phi(\cdot, \tau)\|_{L^{\infty}\left(\mathbb{R}^{N}\right)} \\
=\kappa \tilde{C}_{0}\left(e^{\tilde{\epsilon}_{0}}-1\right)\|\phi(\cdot, \tau)\|_{L^{\infty}\left(\mathbb{R}^{N}\right)}>0 \\
\left(|x| \leq R, t \in\left[\tau+3, \tau+3+T_{0}\right)\right] .
\end{gathered}
$$

Next, the positivity of $\phi$ in $B_{R} \times\left[\tau+3, \tau+3+T_{0}\right]$ and the maximum principle (Lemma 3.5 applied to $-\phi$ ) give

$$
\left\|\phi^{-}\left(\cdot, \tau+T_{0}\right)\right\|_{L^{\infty}\left(\mathbb{R}^{N}\right)} \leq\left\|\phi^{-}(\cdot, \tau+3)\right\|_{L^{\infty}\left(\mathbb{R}^{N}\right)} .
$$

As above (see (6.8)), we also have

$$
\begin{aligned}
\left\|\phi^{-}(\cdot, \tau+3)\right\|_{L^{\infty}\left(\mathbb{R}^{N}\right)} & \leq\left\|u\left(\cdot, \tau+3 ; \tau, \phi^{-}(\cdot, \tau)\right)\right\|_{L^{\infty}\left(\mathbb{R}^{N}\right)} \\
& \leq e^{3 d_{0}}\left\|\phi^{-}(\cdot, \tau)\right\|_{L^{\infty}\left(\mathbb{R}^{N}\right)} .
\end{aligned}
$$

Now, taking $s=\tau+T_{0}$ in (6.12) and using successively (6.3), (6.15), and (6.14), we obtain

$$
\begin{aligned}
\left\|\phi^{+}\left(\cdot, \tau+T_{0}\right)\right\|_{L^{\infty}\left(\mathbb{R}^{N}\right)} & \geq \tilde{C}_{0} e^{\tilde{\epsilon}_{0} T_{0}}\left\|\phi^{+}(\cdot, \tau)\right\|_{L^{\infty}\left(\mathbb{R}^{N}\right)} \\
& \geq \tilde{C}_{0} e^{\tilde{\epsilon}_{0} T_{0}} \alpha\left\|\phi^{-}(\cdot, \tau)\right\|_{L^{\infty}\left(\mathbb{R}^{N}\right)} \\
& \geq \tilde{C}_{0} e^{\tilde{\epsilon}_{0} T_{0}-3 d_{0}} \alpha\left\|\phi^{-}(\cdot, \tau+3)\right\|_{L^{\infty}\left(\mathbb{R}^{N}\right)} \\
& \geq \tilde{C}_{0} e^{\tilde{\epsilon}_{0} T_{0}-3 d_{0}} \alpha\left\|\phi^{-}\left(\cdot, \tau+T_{0}\right)\right\|_{L^{\infty}\left(\mathbb{R}^{N}\right)}
\end{aligned}
$$

Then our choice of $T_{0}$ (see (6.5)) and (6.16) imply

$$
\left\|\phi^{+}\left(\cdot, \tau+T_{0}\right)\right\|_{L^{\infty}\left(\mathbb{R}^{N}\right)} \geq \alpha\left\|\phi^{-}\left(\cdot, \tau+T_{0}\right)\right\|_{L^{\infty}\left(\mathbb{R}^{N}\right)} .
$$

Repeating the above argument with $\alpha$ fixed as in (6.10) and with $\tau$ replaced by $\tau+T_{0}$, we obtain inequalities (6.13) and (6.17) with $\tau$ replaced by $\tau+T_{0}$. Continuing in this way indefinitely, it is easy to conclude that

$$
\phi(x, t)>0 \quad(|x| \leq R, t \in[\tau+3, \infty)) .
$$


We are ready to prove (6.4). Fix any $x \in B_{\rho_{0}}$ and $t \geq \tau+4$. By (6.18) and (6.6) there exists $x_{0} \in B_{R-1}$ such that $\|\phi(\cdot, t-1 / 2)\|_{L^{\infty}\left(\mathbb{R}^{N}\right)}=\phi\left(x_{0}, t-1 / 2\right)$. By the Harnack inequality we then have

$$
\phi(x, t) \geq C\|\phi(\cdot, t-1 / 2)\|_{L^{\infty}\left(\mathbb{R}^{N}\right)} \quad(|x| \leq R-1),
$$

where $C>0$ depends only on the given quantities. Finally, noting that $R-1 \geq \rho_{0}$ and using Lemma 6.1(ii), we obtain the claim.

The further exposition will be simpler with the following result.

Lemma 6.3. There exist a $C^{2}$-function $\beta:\left[s_{0}, \infty\right) \rightarrow \mathbb{R}$ and constants $c_{4}>0, \theta_{2} \geq \theta_{1}>0$ depending only on the given quantities such that $\theta_{2} \beta \geq$ $\beta^{\prime} \geq \theta_{1} \beta>0$ on $\left[s_{0}, \infty\right)$ and

$$
c_{4}^{-1} \leq \frac{\|\phi(\cdot, t)\|_{L^{\infty}\left(\mathbb{R}^{N}\right)}}{\beta(t)} \leq c_{4} \quad\left(t \geq s_{0}\right) .
$$

Proof. Let $\tau>0$ be defined by $\tilde{C}_{0} e^{\tilde{\epsilon}_{0} \tau}=2$, so that, by (H2) and Proposition 3.4 ,

$$
e^{d_{0} \tau} \geq \frac{\left\|\phi\left(\cdot, s_{0}+(k+1) \tau\right)\right\|_{L^{\infty}\left(\mathbb{R}^{N}\right)}}{\left\|\phi\left(\cdot, s_{0}+k \tau\right)\right\|_{L^{\infty}\left(\mathbb{R}^{N}\right)}} \geq 2
$$

for any integer $k \geq 0$. It is then easy to find a $C^{2}$-function $\eta$ such that $\eta\left(s_{0}+k \tau\right)=\ln \left\|\phi\left(\cdot, s_{0}+k \tau\right)\right\|_{L^{\infty}\left(\mathbb{R}^{N}\right)}$ for each $k \in \mathbb{Z}$ and the slope of $\eta$ is in the interval $\left[\ln 2 / \tau, d_{0}\right]$ everywhere. Then, obviously, $\beta:=e^{\eta}$ satisfies $\theta_{2} \beta \geq \beta^{\prime} \geq \theta_{1} \beta>0$ with $\theta_{1}=\ln 2 / \tau$ and $\theta_{2}=d_{0}$. Note that $\tau$ and hence $\theta_{i}$, $i=1,2$, depend only on the given quantities. We claim that this function also satisfies (6.19). Indeed, given any integer $k \geq 0$ and $t \in\left[s_{0}+k \tau, s_{0}+(k+1) \tau\right]$, we use (6.2) and the monotonicity of $\beta$ to obtain

$$
\left(c_{2}(\tau)\right)^{-1}\|\phi(\cdot, t)\|_{L^{\infty}\left(\mathbb{R}^{N}\right)} \leq\left\|\phi\left(\cdot, s_{0}+k \tau\right)\right\|_{L^{\infty}\left(\mathbb{R}^{N}\right)}=\beta\left(s_{0}+k \tau\right) \leq \beta(t)
$$

and

$$
\begin{aligned}
\beta(t) & \leq \beta\left(s_{0}+(k+1) \tau\right) \\
& =\left\|\phi\left(\cdot, s_{0}+(k+1) \tau\right)\right\|_{L^{\infty}\left(\mathbb{R}^{N}\right)} \leq c_{2}(\tau)\|\phi(\cdot, t)\|_{L^{\infty}\left(\mathbb{R}^{N}\right)} .
\end{aligned}
$$

Since $\tau$ is determined by $\tilde{C}_{0}$ and $\tilde{\epsilon}_{0}, c_{4}:=c_{2}(\tau)$ depends only on the given quantities. Thus $\beta$ has all the desired properties. 
It will be convenient in the following to rescale the equation (1.1) by $\beta(t)$, where $\beta$ is the function given by Lemma 6.3. Obviously, $u$ is a solution of $(1.1)$ on $(t, \infty), t \geq s_{0}$, if and only if $\tilde{u}$ defined by $\tilde{u}(\cdot, s)=u(\cdot, s) / \beta(s), s>t$, is a solution of the following equation

$$
u_{t}=\Delta u+\tilde{a} u
$$

where

$$
\tilde{a}(x, t)=a(x, t)-\frac{\beta^{\prime}(t)}{\beta(t)} \quad\left(t \geq s_{0}\right) .
$$

Below we will use the fact that by (A) and the properties of $\beta$ (see Lemma 6.3) one has

$$
\tilde{a}(x, t) \leq-\theta_{1}<0 \quad\left(|x| \geq \rho_{0}, t \geq s_{0}\right), \quad\|\tilde{a}\|_{L^{\infty}\left(\mathbb{R}^{N} \times \mathbb{R}\right)} \leq d_{0}+\theta_{2} .
$$

With $T_{0}$ and $\alpha$ as in Lemma 6.2 define the following numbers

$$
\begin{aligned}
& T_{1}^{+}:=\inf \left\{\tau \geq s_{0}+T_{0}:\left\|\phi^{+}(\cdot, \tau)\right\|_{L^{\infty}\left(\mathbb{R}^{N}\right)} \geq \alpha\left\|\phi^{-}(\cdot, \tau)\right\|_{L^{\infty}\left(\mathbb{R}^{N}\right)}\right\}, \\
& T_{1}^{-}:=\inf \left\{\tau \geq s_{0}+T_{0}:\left\|\phi^{-}(\cdot, \tau)\right\|_{L^{\infty}\left(\mathbb{R}^{N}\right)} \geq \alpha\left\|\phi^{+}(\cdot, \tau)\right\|_{L^{\infty}\left(\mathbb{R}^{N}\right)}\right\},
\end{aligned}
$$

where we define the infimum to be $\infty$ if the set is empty. By Lemma 6.2, replacing $\phi$ by $-\phi$ if necessary, we may assume $T_{1}^{+} \leq T_{1}^{-}=\infty$ (so that the set in (6.24) is empty) and we then have

$$
\frac{1}{\alpha+1} \leq \frac{\left\|\phi^{+}(\cdot, \tau)\right\|_{L^{\infty}\left(\mathbb{R}^{N}\right)}}{\|\phi(\cdot, \tau)\|_{L^{\infty}\left(\mathbb{R}^{N}\right)}} \leq 1 \quad\left(\tau \in\left[s_{0}+T_{0}, \infty\right)\right) .
$$

The proof of Theorem 2.4 uses a family of subsolutions of (6.20) as given in the following lemma.

Lemma 6.4. For each $\epsilon_{0}<\tilde{\epsilon}_{0}$ there exist $R_{\epsilon_{0}}>\rho_{0}, T_{\epsilon_{0}}>T_{0}+1$, depending only on $\tilde{\epsilon}_{0}-\epsilon_{0}$ and the given quantities such that the following holds. For each $t_{0} \geq s_{0}+T_{\epsilon_{0}}$ there exists a subsolution $\tilde{u} \in C\left(\bar{B}_{R_{\epsilon_{0}}} \times\left[t_{0}, \infty\right)\right)$ of $(6.20)$ on $B_{R_{\epsilon_{0}}} \times\left(t_{0}, \infty\right)$ with the following properties:

(i) For some $c_{5}>0$ depending only on the given quantities one has

$$
\frac{\left\|\tilde{u}^{+}(\cdot, t)\right\|_{L^{\infty}\left(B_{R_{0}}\right)}}{\left\|\tilde{u}^{+}\left(\cdot, t_{0}\right)\right\|_{L^{\infty}\left(B_{R_{\epsilon_{0}}}\right)}} \geq c_{5} e^{\left(\epsilon_{0}-\tilde{\epsilon}_{0}\right)\left(t-t_{0}\right)} \quad\left(t \geq t_{0}\right) ;
$$


(ii) $\tilde{u}(x, t)=0$ for $|x|=R_{\epsilon_{0}}, t \geq t_{0}$.

Before proving the lemma, we show how it implies Theorem 2.4.

Proof of Theorem 2.4. Assume that $s_{0}$ and $\phi$ satisfy the hypotheses of the theorem and fix $\epsilon_{0}<\tilde{\epsilon}_{0}$. We prove that (2.11) holds for suitable $R_{0}, C_{0}$. Let $R_{\epsilon_{0}}, T_{\epsilon_{0}}$ be as in Lemma 6.4 and, given any $t \geq s \geq s_{0}+T_{\epsilon_{0}}$, let $\tilde{u}$ be as in Lemma 6.4 with $t_{0}=s$. Taking $R=R_{\epsilon_{0}}+1$ in (4.3), we have

$$
\left\|\varphi_{R_{\epsilon_{0}}+1}(\cdot, t)\right\|_{L^{\infty}\left(B_{R_{\epsilon_{0}}+1}\right)} \leq \tilde{C}\left(R_{\epsilon_{0}}\right) \inf _{x \in B_{R_{\epsilon_{0}}}} \varphi_{R_{\epsilon_{0}}+1}(x, t) \quad(t \in \mathbb{R}),
$$

where $\tilde{C}\left(R_{\epsilon_{0}}\right)>0$ depends only on $R_{\epsilon_{0}}$ and the given quantities. Using this in conjunction with the Harnack inequality and maximum principle, we find a positive constant $C\left(\epsilon_{0}\right)$, depending only on $\tilde{\epsilon}_{0}-\epsilon_{0}$ and the given quantities, such that

$$
C^{-1}\left(\epsilon_{0}\right) \leq \frac{\left\|\varphi_{R_{\epsilon_{0}}+1}\left(\cdot, \tau_{1}\right)\right\|_{L^{\infty}\left(B_{R_{\epsilon_{0}}+1}\right)}}{\left\|\varphi_{R_{\epsilon_{0}}+1}\left(\cdot, \tau_{2}\right)\right\|_{L^{\infty}\left(B_{R_{\epsilon_{0}}+1}\right)}} \leq C\left(\epsilon_{0}\right) \quad\left(\tau_{1}, \tau_{2} \in \mathbb{R},\left|\tau_{1}-\tau_{2}\right| \leq T_{\epsilon_{0}}\right) .
$$

Set $\tilde{\varphi}_{R_{\epsilon_{0}}+1}=\varphi_{R_{\epsilon_{0}}+1} / \beta$, where $\beta$ is as in Lemma 6.3. Then $\tilde{\varphi}_{R_{\epsilon_{0}}+1}$ is a positive entire solution of (6.20) on $B_{R_{\epsilon_{0}}+1} \times \mathbb{R}$ vanishing on $\partial B_{R_{\epsilon_{0}}+1} \times \mathbb{R}$ and (6.27) and (6.28) still hold if we replace $\varphi_{R_{\epsilon_{0}}+1}$ by $\tilde{\varphi}_{R_{\epsilon_{0}}+1}$.

For any $x \in B_{R_{\epsilon_{0}}}$

$$
\begin{aligned}
\tilde{\varphi}_{R_{\epsilon_{0}}+1}(x, s) & \geq \tilde{C}^{-1}\left(R_{\epsilon_{0}}\right)\left\|\tilde{\varphi}_{R_{\epsilon_{0}}+1}(\cdot, s)\right\|_{L^{\infty}\left(B_{R_{\epsilon_{0}}+1}\right)}=k\left\|\tilde{u}^{+}(\cdot, s)\right\|_{L^{\infty}\left(B_{R_{\epsilon_{0}}}\right)} \\
& \geq k \tilde{u}(x, s),
\end{aligned}
$$

where the constant $k$ is chosen such that the equation holds. Since the subsolution $\tilde{u}$ is equal to zero on $\partial B_{R_{\epsilon_{0}}} \times[s, \infty)$, a comparison argument gives $\tilde{\varphi}_{R_{\epsilon_{0}}+1} \geq k \tilde{u}$ in $B_{R_{\epsilon_{0}}} \times[s, \infty)$. Consequently, by (6.29), (6.26),

$$
\frac{\left\|\tilde{\varphi}_{R_{\epsilon_{0}}+1}(\cdot, t)\right\|_{L^{\infty}\left(B_{R_{\epsilon_{0}}+1}\right)}}{\left\|\tilde{\varphi}_{R_{\epsilon_{0}}+1}(\cdot, s)\right\|_{L^{\infty}\left(B_{R_{\epsilon_{0}}+1}\right)}} \geq \frac{\left\|\tilde{u}^{+}(\cdot, t)\right\|_{L^{\infty}\left(B_{R_{\epsilon_{0}}}\right)}}{C\left(R_{\epsilon_{0}}\right)\left\|\tilde{u}_{+}(\cdot, s)\right\|_{L^{\infty}\left(B_{R_{\epsilon_{0}}}\right)}} \geq \frac{c_{5}}{C\left(R_{\epsilon_{0}}\right)} e^{\left(\epsilon_{0}-\tilde{\epsilon}_{0}\right)(t-s)} .
$$

This estimate together with (6.28) (applied to $\tilde{\varphi}_{R_{\epsilon_{0}}+1}$ ) imply that the above inequality holds for any $t \geq s \geq s_{0}$ with a possibly smaller constant replacing $c_{5} / C\left(R_{\epsilon_{0}}\right)$ but still depending only on $\tilde{\epsilon}_{0}-\epsilon_{0}$ and the given quantities. Using Lemma 6.3 and the assumption (2.3), we get

$$
\frac{\beta(t)}{\beta(s)} e^{-\tilde{\epsilon}_{0}(t-s)} \geq \frac{\tilde{C}_{0}}{c_{4}^{2}}
$$


The last two inequalities and the definition of $\tilde{\varphi}_{R_{\epsilon_{0}}+1}$ then imply

$$
\frac{\left\|\varphi_{R_{\epsilon_{0}}+1}(\cdot, t)\right\|_{L^{\infty}\left(B_{R_{\epsilon_{0}}+1}\right)}}{\left\|\varphi_{R_{\epsilon_{0}}+1}(\cdot, s)\right\|_{L^{\infty}\left(B_{R_{\epsilon_{0}}+1}\right)}} \geq C_{0} e^{\epsilon_{0}(t-s)} \quad\left(t \geq s \geq s_{0}\right)
$$

for some $C_{0}>0$ depending only on $\tilde{\epsilon}_{0}-\epsilon_{0}$ and the given quantities. Taking $R_{0}=R_{\epsilon_{0}}+1$ we have thus proved the desired estimate (2.11).

It remains to prove Lemma 6.4.

Proof of Lemma 6.4. Fix an arbitrary $\epsilon_{0}<\tilde{\epsilon}_{0}$. Define $\tilde{\phi}=\phi / \beta$, where $\beta$ is as in Lemma 6.3. Then, as noted above, $\phi$ is a solution of (6.20) on $\mathbb{R}^{N} \times\left(s_{0}, \infty\right)$. Using Lemma 6.3 and $(6.25)$ we obtain

$$
\frac{1}{(\alpha+1) c_{4}} \leq\left\|\tilde{\phi}^{+}(\cdot, t)\right\|_{L^{\infty}\left(\mathbb{R}^{N}\right)} \leq\|\tilde{\phi}(\cdot, t)\|_{L^{\infty}\left(\mathbb{R}^{N}\right)} \leq c_{4} \quad\left(t \geq s_{0}+T_{0}\right) .
$$

These inequalities together with (6.1) guarantee the existence of $R \geq \rho_{0}+1$, $T \geq T_{0}$ depending only on the given quantities such that

$$
\frac{1}{(\alpha+1) c_{4}} \leq\left\|\tilde{\phi}^{+}(\cdot, t)\right\|_{L^{\infty}\left(B_{R}\right)}=\left\|\tilde{\phi}^{+}(\cdot, t)\right\|_{L^{\infty}\left(\mathbb{R}^{N}\right)} \leq c_{4} \quad\left(t \geq s_{0}+T\right) .
$$

Again using (6.1) and (6.31), it is easy to show that for each $\delta>0$ there exist $R(\delta) \geq R+1, T(\delta) \geq T$ depending only on $\delta$ and the given quantities such that

$$
|\tilde{\phi}(x, t)| \leq \delta \quad\left(|x| \geq R(\delta), t \geq s_{0}+T(\delta)\right) .
$$

We choose $\delta>0$ sufficiently small so that

$$
\delta<\min \left\{1, \frac{1}{(\alpha+1) c_{4}}\right\}
$$

and another condition specified below is satisfied. For the corresponding $R(\delta), T(\delta)$, set $t_{0}=s_{0}+T(\delta)+1$ and let $w^{\delta}$ be the solution of the following initial-boundary value problem

$$
\begin{aligned}
u_{t} & =\Delta u+\tilde{a}(x, t) u \quad \text { in } \quad B_{R(\delta)} \times\left(t_{0}-1, \infty\right), \\
u & =\delta \quad \text { on } \quad \partial B_{R(\delta)} \times\left(t_{0}-1, \infty\right), \\
u\left(x, t_{0}-1\right) & =\max \left\{\tilde{\phi}^{+}\left(x, t_{0}-1\right), \delta\right\} \quad x \in B_{R(\delta)} .
\end{aligned}
$$


That such a solution exists is guaranteed by standard existence theory (see [19]) and the fact that $\max \left\{\tilde{\phi}^{+}\left(\cdot, t_{0}-1\right), \delta\right\}$ is a continuous function equal to $\delta$ on $\partial B_{R(\delta)}$.

With $\theta_{1}$ as in Lemma 6.3, set $\nu:=\min \left\{\theta_{1}, \tilde{\epsilon}_{0}-\epsilon_{0}\right\}$ and

$$
\tilde{u}(x, t):=e^{-\nu t}\left(w^{\delta}(x, t)-\delta\right) \quad\left(x \in B_{R(\delta)}, t \geq t_{0}\right) .
$$

We show that $\tilde{u}$ has the stated properties. For this goal we first collect some properties of the solution $w^{\delta}$.

By $(6.33),(6.35), w^{\delta}$ dominates $\tilde{\phi}^{+}$on the parabolic boundary of $B_{R(\delta)} \times$ $\left(t_{0}-1, \infty\right)$. Therefore, by the comparison principle

$$
w^{\delta}(x, t) \geq \tilde{\phi}^{+}(x, t) \quad\left(x \in B_{R(\delta)}, t \geq t_{0}-1\right) .
$$

Thus, by (6.32) and the Harnack inequality we have

$$
\inf _{x \in B_{\rho_{0}}} w^{\delta}(x, t) \geq C>0 \quad\left(t \geq t_{0}\right),
$$

where $C$ depends only on the given quantities. In view of (6.34) and (6.31), applying the maximum principle we obtain

$$
\left\|w^{\delta}\left(\cdot, t_{0}\right)\right\|_{L^{\infty}\left(B_{R(\delta)}\right)} \leq \tilde{C},
$$

with $\tilde{C}>0$ depending only on the given quantities.

Next we verify that $\tilde{u}$ is a subsolution of $(6.20)$ on $B_{R(\delta)} \times\left(t_{0}, \infty\right)$. For any $(x, t)$ in that set we have (omitting the argument $(x, t)$ of $w^{\delta}$ for brevity)

$$
\begin{aligned}
& \tilde{u}_{t}(x, t)-\Delta \tilde{u}(x, t)-\tilde{a}(x, t) \tilde{u}(x, t) \\
& \quad=e^{-\nu t}\left(-\nu\left(w^{\delta}-\delta\right)+w_{t}^{\delta}-\Delta w^{\delta}-\tilde{a}(x, t) w^{\delta}+\delta \tilde{a}(x, t)\right) \\
& \quad=e^{-\nu t}\left(-\nu\left(w^{\delta}-\delta\right)+\delta \tilde{a}(x, t)\right) .
\end{aligned}
$$

Since $w^{\delta} \geq 0,(6.22)$ and our choice of $\nu$ imply that the last expression is nonpositive, for any $\delta>0$, if $|x| \geq \rho_{0}$. On the other hand, if $|x|<\rho_{0}$, then by (6.37) and the bound on $\tilde{a}$ (see (6.22)) this expression is nonpositive whenever $\delta \leq \min \left\{C / 2, \nu C /\left(2\left(d_{0}+\theta_{2}\right)\right)\right\}$. One can therefore choose $\delta>0$ depending only on $\tilde{\epsilon}_{0}-\epsilon_{0}$ and the given quantities such that (in addition to (6.34)) $\tilde{u}$ is a subsolution of $(6.20)$ on $B_{R(\delta)} \times\left(t_{0}, \infty\right)$. Fix such $\delta$ and denote $R_{\epsilon_{0}}=R(\delta), T_{\epsilon_{0}}=T(\delta)$. Note that these numbers depend only on $\tilde{\epsilon}_{0}-\epsilon_{0}$ and the given quantities. It remains to verify $(i)$ and $(i i)$ in Lemma 6.4. 
Obviously, by the definitions of $w^{\delta}$ and $\tilde{u}$, we have $\tilde{u}=0$ for $|x|=R_{\epsilon_{0}}$, $t \geq t_{0}$, verifying $(i i)$.

To verify $(i)$, note that $\nu \leq \tilde{\epsilon}_{0}-\epsilon_{0}$ and $\delta \leq C / 2$, where $C$ is as in (6.37). Using this, (6.37) and (6.38) we get for any $t \geq t_{0}$

$$
\begin{aligned}
\frac{\left\|\tilde{u}^{+}(\cdot, t)\right\|_{L^{\infty}\left(B_{R_{\epsilon_{0}}}\right)}}{\left\|\tilde{u}^{+}\left(\cdot, t_{0}\right)\right\|_{L^{\infty}\left(B_{R_{0}}\right)}} & \geq e^{-\nu\left(t-t_{0}\right)} \frac{\left\|w^{\delta}(\cdot, t)\right\|_{L^{\infty}\left(B_{R_{\epsilon_{0}}}\right)}-\delta}{\left\|w^{\delta}\left(\cdot, t_{0}\right)\right\|_{L^{\infty}\left(B_{R_{\epsilon_{0}}}\right)}+\delta} \\
& \geq e^{-\nu\left(t-t_{0}\right)} \frac{C}{2 \tilde{C}+C} \geq e^{\left(\epsilon_{0}-\tilde{\epsilon}_{0}\right)\left(t-t_{0}\right)} \frac{C}{2 \tilde{C}+C} .
\end{aligned}
$$

Hence estimate (6.26) holds with $c_{5}=C /(2 \tilde{C}+C)$.

\section{Proofs of Proposition 2.5 and Theorem 2.6}

Proof of Proposition 2.5. Using the notation as in Theorem 2.2, we first choose $q \in \mathbb{R}$ such that $u:=\tilde{\varphi}-q \varphi$ satisfies $u(\cdot, 0) \in X_{2}(0)$, that is, $\int_{\mathbb{R}^{N}} u(x, 0) \psi(x, 0) d x=0$. By Lemma 5.1, we then have $u(\cdot, t) \in X_{2}(t)$ for all $t \in \mathbb{R}$. We show that $u \equiv 0$.

Assume not. Then $u\left(t_{0}\right) \not \equiv 0$ for some $t_{0}$ (in fact, this is true for any $t_{0}$ by backward uniqueness), and using the exponential separation we obtain

$$
\frac{\|\tilde{\varphi}(\cdot, t)\|_{L^{\infty}\left(\mathbb{R}^{N}\right)}}{\|\varphi(\cdot, t)\|_{L^{\infty}\left(\mathbb{R}^{N}\right)}}+q \geq \frac{\|u(\cdot, t)\|_{L^{\infty}\left(\mathbb{R}^{N}\right)}}{\|\varphi(\cdot, t)\|_{L^{\infty}\left(\mathbb{R}^{N}\right)}} \geq C e^{\gamma\left(t_{0}-t\right)} \frac{\left\|u\left(\cdot, t_{0}\right)\right\|_{L^{\infty}\left(\mathbb{R}^{N}\right)}}{\left\|\varphi\left(\cdot, t_{0}\right)\right\|_{L^{\infty}\left(\mathbb{R}^{N}\right)}} \rightarrow \infty
$$

as $t \rightarrow-\infty$. Since $\|\tilde{\varphi}(\cdot, t)\|_{L^{\infty}\left(\mathbb{R}^{N}\right)} \leq c_{1}\|\tilde{\varphi}(\cdot, t-1)\|_{L^{\infty}\left(B_{R}\right)}$, by assumption (2.12) and Proposition 3.4, we also have

$$
\frac{\|\tilde{\varphi}(\cdot, t-1)\|_{L^{\infty}\left(B_{R}\right)}}{\|\varphi(\cdot, t)\|_{L^{\infty}\left(\mathbb{R}^{N}\right)}} \rightarrow \infty .
$$

Using this and the Harnack inequality, we conclude that

$$
\min _{x \in B_{R}} \frac{\tilde{\varphi}(x, t)}{\|\varphi(\cdot, t)\|_{L^{\infty}\left(\mathbb{R}^{N}\right)}}
$$

converges to $\infty$ and hence also

$$
\int_{B_{R}} \frac{\tilde{\varphi}(x, t)}{\|\varphi(\cdot, t)\|_{L^{\infty}\left(\mathbb{R}^{N}\right)}} d x \rightarrow \infty
$$


On the other hand, by Lemma 5.3,

$$
\begin{aligned}
\int_{B_{R}} \frac{\tilde{\varphi}(x, t)}{\|\varphi(\cdot, t)\|_{L^{\infty}\left(\mathbb{R}^{N}\right)}} d x & \leq C_{1} \int_{B_{R}} \frac{\tilde{\varphi}(x, t)}{\|\varphi(\cdot, t)\|_{L^{\infty}\left(\mathbb{R}^{N}\right)}} \frac{\psi(x, t)}{\|\psi(\cdot, t)\|_{L^{\infty}\left(\mathbb{R}^{N}\right)}} d x \\
& \leq C_{2} \int_{B_{R}} \tilde{\varphi}(x, t) \psi(x, t) d x \leq C_{2} \int_{\mathbb{R}^{N}} \tilde{\varphi}(x, t) \psi(x, t) d x,
\end{aligned}
$$

for some constants $C_{1}, C_{2}$. By Lemma 5.1 the last integral is independent of $t$ and we have a contradiction to (7.1).

Proof of Theorem 2.6. Fix any $\epsilon<\tilde{\epsilon}_{0}$ and set $\epsilon_{1}=\tilde{\epsilon}_{0}-\epsilon$. Applying Theorem 2.4 with $\epsilon_{0}=\tilde{\epsilon}_{0}-\epsilon_{1} / 4$, we find positive constants $C_{0}, R_{0}$ depending only on the given quantities $\left(N, d_{0}, \rho_{0}, \tilde{C}_{0}, \tilde{\epsilon}_{0}\right)$ and $\epsilon_{1}$ such that $(2.11)$ holds for each $s_{0} \in \mathbb{R}$. We now use a robustness result for (2.1). For that we denote by $\tilde{\varphi}_{R_{0}}$ the positive entire solution of (2.1) with $a$ replaced by $\tilde{a}$ (assuming the usual normalization $\left.\left\|\tilde{\varphi}_{R}(\cdot, 0)\right\|_{L^{\infty}\left(B_{R}\right)}=1\right)$. Then by Proposition 8.3 of $[16]$ there exist positive constants $\delta$ and $C_{1}$ depending only on $\epsilon_{1}$ and the given quantities such that if $\|a-\tilde{a}\|_{L^{\infty}\left(\mathbb{R}^{N} \times \mathbb{R}\right)}<\delta$ then

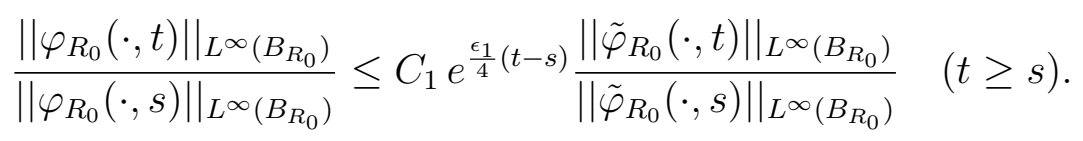

Combining this with (2.11), we obtain

$$
\frac{\left\|e^{\frac{-\epsilon_{1}}{2} t} \tilde{\varphi}_{R_{0}}(\cdot, t)\right\|_{L^{\infty}\left(B_{R_{0}}\right)}}{\left\|e^{\frac{-\epsilon_{1}}{2} s} \tilde{\varphi}_{R_{0}}(\cdot, s)\right\|_{L^{\infty}\left(B_{R_{0}}\right)}} \geq C_{1}^{-1} C_{0} e^{\epsilon(t-s)} \quad(t \geq s) .
$$

Finally, we transform equation (2.13) by $u \rightarrow e^{-\epsilon_{1} t / 2} u$ to obtain

$$
u_{t}=\Delta u+\left(\tilde{a}(x, t)-\frac{\epsilon_{1}}{2}\right) u \quad \text { in } \quad \mathbb{R}^{N} \times J .
$$

If $\delta$ is greater than $\epsilon_{1} / 2$ we replace it by $\epsilon_{1} / 2$ and then $\|a-\tilde{a}\|_{L^{\infty}\left(\mathbb{R}^{N}\right)}<\delta$ guarantees that the coefficient in (7.3) is nonpositive outside $B_{\rho_{0}}$. Further, $e^{\frac{-\epsilon_{1}}{2} t} \tilde{\varphi}_{R_{0}}(\cdot, t)$ is the positive entire solution of $(2.1)$ with $a$ replaced by $\tilde{a}-\epsilon_{1} / 2$. Hence, by (7.2), Theorem 2.1 applies to (7.3), which readily implies the conclusion of Theorem 2.6. 


\section{A remark on the hypotheses}

In this section we show that even if hypothesis (A) holds in the strict sense $\left(a<0\right.$ outside a ball), condition (H2) cannot be relaxed by allowing $\tilde{\epsilon}_{0}=0$ and requiring instead that the solution $\phi$ decay exponentially as $|x| \rightarrow \infty$. Counterexamples exist even in the autonomous case.

Proposition 8.1. There exists a bounded radially symmetric function $a=$ $a(|x|)$ with the following properties:

(i) for some $\rho_{0}>0$ one has $a(|x|)<0 \quad\left(|x| \geq \rho_{0}\right)$,

(ii) there exists a radially symmetric positive solution $\varphi$ of the equation $\Delta \varphi+a(|x|) \varphi=0$ on $\mathbb{R}^{N}$ satisfying for some $c>0$ the following estimate

$$
\varphi(x) \leq(1 / c) e^{-c|x|} \quad\left(x \in \mathbb{R}^{N}\right) .
$$

(iii) $\sigma(\Delta+a(x))=(-\infty, 0]$, where $\Delta+a(x)$ is viewed as an operator on $L^{2}\left(\mathbb{R}^{N}\right)$.

Note that for such $a$ the conclusion of Theorem 2.2 (with $\varphi(x, t)=$ $\psi(x, t)=\varphi(x))$ cannot hold. Indeed, $\varphi$ is an eigenfunction of $\Delta+a(|x|)$ with eigenvalue 0 . The conclusion of Theorem 2.2 (see also Remark 2.3) would imply that 0 is an isolated eigenvalue in contradiction to (iii). The proposition also provides another example of an operator $\Delta+a(|x|)$ which has an embedded eigenvalue with exponentially decaying eigenfunction (an example with a periodic $a$ is given [8]).

Proof of Proposition 8.1. Define

$$
a(r)=1+\frac{N-1}{r} \frac{\sin (r)}{\cos (r)} \quad(r \in(0, \pi / 4)),
$$

and

$$
a(r)=-\eta^{2}(r)-\frac{N-1}{r} \eta(r)-\eta^{\prime}(r) \quad(r \geq \pi / 4),
$$

where the function $\eta:[\pi / 4, \infty) \rightarrow[-1,0)$ is to be defined below in such a way that

$$
\begin{aligned}
& \eta \in C^{1}[\pi / 4, \infty), \quad \eta(\pi / 4)=-1, \\
& a \in L^{\infty}(0, \infty), \quad a(r)<0 \text { for all sufficiently large } r
\end{aligned}
$$


Defining further

$$
\begin{aligned}
& \varphi(x)=\cos (|x|) \quad(|x|<\pi / 4), \\
& \varphi(x)=\frac{\sqrt{2}}{2} e^{\int_{\frac{\pi}{4}}^{|x|} \eta(r) d r} \quad(|x| \geq \pi / 4),
\end{aligned}
$$

it is easy to check that $\varphi$ is a positive $C^{1}$-function satisfying $\Delta \varphi(x)+$ $a(|x|) \varphi(x)=0$ for all $x \in \mathbb{R}^{N} \backslash\{|x|=\pi / 4\}$ and hence it is a positive solution of this equation.

The proof will be completed if we define a function $\eta$ in such a way that (8.4) and statements (i), (iii) of Proposition 8.1 hold. and

We define sequences $\left\{a_{i}\right\}_{i=1}^{\infty},\left\{b_{i}\right\}_{i=1}^{\infty},\left\{c_{i}\right\}_{i=1}^{\infty},\left\{d_{i}\right\}_{i=1}^{\infty}$ by setting $a_{1}=\pi / 4$

$$
b_{i}=2 a_{i}, \quad c_{i}=b_{i}+i^{4}, \quad d_{i}=c_{i}+i, \quad a_{i+1}=d_{i}+i^{4}, \quad(i=1,2, \ldots) .
$$

Obviously $0<a_{i}<b_{i}<c_{i}<d_{i}<a_{i+1}$. Set

$$
\begin{array}{ll}
\eta(r)=-1 & \left(r \in\left[a_{i}, b_{i}\right)\right), \\
\eta(r)=\frac{-1}{2 i} & \left(r \in\left[c_{i}, d_{i}\right)\right) .
\end{array}
$$

Observe that (8.3), (8.8), (8.9), and the fact that $c_{i} \geq 2^{i-3} \pi$ (which follows from (8.7)) ensure that $a(r)<0$ for all $r$ sufficiently large belonging to the intervals $\left[a_{i}, b_{i}\right)$ or $\left[c_{i}, d_{i}\right)$.

Next we extend $\eta$ by

$$
\eta(r)=\frac{-1}{2 i}-e^{-1 /\left(r-d_{i}\right)} \quad\left(r \in\left[d_{i}, d_{i}+\delta_{i}\right]\right),
$$

where $\delta_{i}=1 / \ln \left(i^{4}\right)$. Note that the extension is $C^{1}, \eta\left(d_{i}\right)=-1 /(2 i)$, and $\eta^{\prime}\left(d_{i}\right)=0$. It is also easy to check that $a(r)<0$ for all $r \in\left[d_{i}, d_{i}+\delta_{i}\right]$ and $i$ large enough, and since $\eta^{\prime}\left(d_{i}+\delta_{i}\right)=-16(\ln (i))^{2} / i^{4}$, we can further extend $\eta$ to the interval $\left[d_{i}, a_{i+1}\right]$ in a $C^{1}$ manner so that $\eta$ is decreasing, $\eta\left(a_{i+1}\right)=-1, \eta^{\prime}\left(a_{i+1}\right)=0$, and $a(r)<0$ for all $r \in\left[d_{i}, a_{i+1}\right]$. Indeed, this can be achieved by first continuing $\eta$ as a linear function on $\left[d_{i}+\delta_{i}, \tilde{d}_{i}\right]$ (with the slope equal to $\left.-16(\ln (i))^{2} / i^{4}\right)$ and then "rounding it off" in a smooth decreasing manner on the interval $\left[\tilde{d}_{i}, a_{i+1}\right]\left(\tilde{d}_{i}\right.$ is a suitably chosen number in $\left.\left[d_{i}, a_{i+1}\right]\right)$. We also note that thus defined function $\eta$ gives rise to a bounded function $a$ on $\left[d_{i}, a_{i+1}\right]$ and the bound is independent of $i$. Finally, we define 
$\eta(r)=\eta\left(d_{i}+c_{i}-r\right)$ whenever $r \in\left[b_{i}, c_{i}\right)$. The definition of $\eta$ is thus complete. It is straightforward to check that $a$ as defined in $(8.2),(8.3)$ is in $L^{\infty}(0, \infty)$ and is negative outside a sufficiently large ball $B_{\rho_{0}}$. Note that (8.7) implies $a_{i}>2^{i-3} \pi$ and $a_{i}<b_{i}=2 a_{i}<a_{i+1}=2 a_{i}+2 i^{4}+i<4 a_{i}$ for all $i$ sufficiently large. This fact, (8.8) and nonpositivity of $\eta$ guarantee the existence of $\tilde{c}>0$ such that $\int_{\frac{\pi}{4}}^{|x|} \eta(r) d r<-\tilde{c}|x|$ for all $x \in \mathbb{R}^{N}$ with $|x| \geq \pi / 2$. This proves that $\varphi$ satisfies (8.1) for some $c>0$.

We prove that $\sigma(\Delta+a(x))=(-\infty, 0]$, where $\Delta+a(x)$ is viewed as an operator on $L^{2}\left(\mathbb{R}^{N}\right)$. By the Kato-Rellich theorem the operator $\Delta+a(x)$ is self-adjoint, therefore $\sigma(\Delta+a(x)) \subset \mathbb{R}$. Further, since $\sigma(\Delta)=(-\infty, 0]$ and $-1 /(2 i)<a(x)<0$ whenever $|x| \in\left[c_{i}, c_{i}+i\right]$ and $i$ is large enough, we have $\sigma(\Delta+a(x)) \supseteq(-\infty, 0]$. Indeed, this is easily proved using approximate eigenfunctions of $\Delta$ (see the proof of [10, Theorem 7.6]). Moreover, as $a(x)<0$ for all large $x$ the essential spectrum of $\Delta+a(x)$ is contained in $(-\infty, 0]$. Finally, since we know that 0 is a an eigenvalue with an exponentially decaying positive eigenfunction, it is must be the largest eigenvalue (see [35]) hence $\sigma(\Delta+a(x))=(-\infty, 0]$, completing the proof of Proposition 8.1 .

\section{Generalizations}

In this section we discuss extensions of our results to more general equations. As we do not want to make the paper significantly longer, we only consider a class of equations for which the results can be proved with only minor modifications of the proofs given above. We consider an equation

$$
u_{t}=L(x, t) u \quad \text { in } \quad \mathbb{R}^{N} \times J
$$

where $L(x, t)$ is a time-dependent second-order elliptic operator in divergence form:

$$
L(x, t) u=\partial_{i}\left(a_{i j}(x, t) \partial_{j} u+a_{i}(x, t) u\right)-b_{i}(x, t) \partial_{i} u+a(x, t) u
$$

(we use the summation convention, and the notation $\partial_{i}=\partial /\left(\partial x_{i}\right)$ ). The adjoint equation to (9.1) is

$$
-v_{t}=L^{*}(x, t) v \quad \text { in } \quad \mathbb{R}^{N} \times J,
$$


where

$$
L^{*}(x, t) v=\partial_{j}\left(a_{j i}(x, t) \partial_{i} v+b_{j}(x, t) v\right)-a_{i}(x, t) \partial_{i} v+a(x, t) v .
$$

We make the assumption (A) on the coefficient $a$ (see Section 2.1) and the following assumptions on the remaining coefficients:

(B) (i) The functions $a_{i j}, a_{i}, b_{i}$ are of class $C_{b}^{1}$ with respect to $x$. This means that these functions and their first order $x$-derivatives are continuous and bounded on $\mathbb{R}^{N} \times \mathbb{R}$. We let $d_{0}$ denote a common bound on all these functions and derivatives, as well as on $a$ (as in (A)). We also assume that for some $\alpha_{0}>0$ the ellipticity condition is satisfied.

$$
a_{j i}(x, t) \xi_{i} \xi_{j} \geq \alpha_{0}|\xi|^{2} \quad\left((x, t) \in \mathbb{R}^{N+1}, \xi=\left(\xi_{1}, \ldots, \xi_{N}\right) \in \mathbb{R}^{N}\right) .
$$

(ii) With $\rho_{0}$ as in (A), one has (using the summation convention again)

$$
\partial_{i} a_{i}(x, t), \partial_{j} b_{j}(x, t) \leq 0 \quad\left(|x| \geq \rho_{0}, t \in \mathbb{R}\right) .
$$

These are rather strong assumptions, but they allow for the simplest proofs of our results; in fact, the proofs are almost identical to those for the model equation (1.1). The point is that with the assumed regularity we can rewrite equation (9.1) in the nondivergence form,

$$
u_{t}=a_{i j}(x, t) \partial_{i} \partial_{j} u+B_{i}(x, t) \partial_{i} u+A(x, t) u,
$$

with $B_{i}, A \in L^{\infty}\left(\mathbb{R}^{N} \times \mathbb{R}\right)$. Hence, similarly as in the case of (1.1), we can take advantage of both forms - the divergence form in applications of the weak Green's function and energy estimates, and the nondivergence form for the maximum principle and construction of sub- and super-solutions. In these constructions, it is also needed that $A(x, t) \leq 0$ for $|x| \geq \rho_{0}$, which is guaranteed by (B)(ii). Similar remarks apply to the adjoint equation (9.2). With more general equations in either divergence or nondivergence form, significantly more involved arguments seem to be necessary.

We remark that one can relax (B)(ii) to require that for some $\rho_{0}^{\prime}$

$$
\partial_{i} a_{i}(x, t), \partial_{j} b_{j}(x, t) \leq \epsilon_{1} \quad\left(|x| \geq \rho_{0}^{\prime}, t \in \mathbb{R}\right),
$$

where $\epsilon_{1}>0$ is sufficiently small (smaller than $\epsilon_{0}$ or $\tilde{\epsilon}_{0}$ in hypotheses (H1)' and (H2)' formulated below). This is satisfied, in particular, if $\partial_{i} a_{i}, \partial_{i} b_{i}$ decay to zero as $|x| \rightarrow \infty$, uniformly in $t$. 
Given $u_{0} \in L^{\infty}\left(\mathbb{R}^{N}\right), s \in \mathbb{R}$, we consider the unique weak solution (in the sense of $[3,19])$ of $(9.1)$ with the initial condition $u(\cdot, s)=u_{0}$. The solution, further denoted by $u\left(x, t ; s, u_{0}\right)$, has again the integral representation (3.4), where $\Gamma(x, t ; y, s)$ is the weak Green's function of (9.1) (cf. [3, Theorem 10]). At the same time, $u\left(x, t ; s, u_{0}\right)$ is a strong solution (in the sense of $[18,20]$ ) on $\mathbb{R}^{N} \times(s, \infty)$, by our regularity assumption.

To reformulate our main results in the more general setting, we introduce similar instability assumptions as in Section 2: (H1)', (H2)' read as (H1), (H2), respectively, with (1.1) replaced by (9.1). Given quantities now refer to $N, d_{0}, \alpha_{0}, \rho_{0}, C_{0}, \epsilon_{0}$ and $R_{0}$ if (A), (B), and (H1)' are assumed, and to $d_{0}, \alpha_{0}, \rho_{0}, \tilde{C}_{0}$, and $\tilde{\epsilon}_{0}$ if $(\mathrm{A}),(\mathrm{B})$, and (H2)' are assumed.

Theorem 9.1. Assume (A), (B) and (H1)'. Then there exist positive entire solutions $\varphi, \psi$ of (9.1) and (9.2), respectively, such that for all $(x, t) \in \mathbb{R}^{N} \times \mathbb{R}$

$$
\frac{\varphi(x, t)}{\|\varphi(\cdot, t)\|_{L^{\infty}\left(\mathbb{R}^{N}\right)}} \leq c_{1} e^{-\nu_{0}|x|}, \frac{\psi(x, t)}{\|\psi(\cdot, t)\|_{L^{\infty}\left(\mathbb{R}^{N}\right)}} \leq c_{1} e^{-\nu_{0}|x|}
$$

and for all $t \geq s$

$$
\frac{\|\varphi(\cdot, t)\|_{L^{\infty}\left(\mathbb{R}^{N}\right)}}{\|\varphi(\cdot, s)\|_{L^{\infty}\left(\mathbb{R}^{N}\right)}} \geq c_{2} e^{\epsilon_{0}(t-s)} \quad \text { and } \quad \frac{\|\psi(\cdot, t)\|_{L^{\infty}\left(\mathbb{R}^{N}\right)}}{\|\psi(\cdot, s)\|_{L^{\infty}\left(\mathbb{R}^{N}\right)}} \leq c_{3} e^{-\epsilon_{0}(t-s)} .
$$

Here $\nu_{0}, c_{1}, c_{2}, c_{3}$ are positive constants depending only on the given quantities.

Note that the only difference, compared to Theorem 2.1, is that we can no longer specify $\nu_{0}=\sqrt{\epsilon_{0}}$ in (9.4).

The exponential separation theorem holds in the same form as for (1.1):

Theorem 9.2. The statement of Theorem 2.2 remains valid if instead of (A), (H1) one assumes (A), (B) and (H1)', and the reference to Theorem 2.1 is replaced by the reference to Theorem 9.1.

As (H1), (H2), the hypotheses (H1)', (H2)' are equivalent:

Theorem 9.3. Assume that (A), (B) and (H2)' hold. Then (H1)' hold.

The last statement can be made more precise as in Theorem 2.4. 
Proposition 9.4. The statement of Proposition 2.5 remains valid if the hypotheses are replaced by (A), (B) and (H1)', or (A), (B) and (H2)', and the references to Theorem 2.1 and equations (1.1), (2.4) are replaced by the corresponding references to Theorem 9.1 and equations (9.1), (9.2).

Finally, we state a theorem on robustness of the instability condition. We formulate it in a slightly different way than Theorem 2.6 so that we only need minimal assumptions on the perturbed equation. We consider the perturbation in the form

$$
u_{t}=\tilde{L}(x, t) u \quad \text { in } \quad \mathbb{R}^{N} \times J,
$$

where

$$
\tilde{L}(x, t) u=\partial_{i}\left(\tilde{a}_{i j}(x, t) \partial_{j} u+\tilde{a}_{i}(x, t) u\right)-\tilde{b}_{i}(x, t) \partial_{i} u+\tilde{a}(x, t) u .
$$

We assume that $\tilde{a}_{i j}, \tilde{a}_{i}, \tilde{b}_{i}, \tilde{a} \in L^{\infty}\left(\mathbb{R}^{N} \times \mathbb{R}\right)$ and that the ellipticity condition (9.3) is satisfied with $a_{i j}$ replaced by $\tilde{a}_{i j}$ and $\alpha_{0}$ replaced by some $\tilde{\alpha}_{0}>0$. For $R>0$ we denote by $\tilde{\varphi}_{R}$ the unique positive entire solution of

$$
\begin{aligned}
u_{t} & =\tilde{L}(x, t) u, \quad \text { in } \quad B_{R} \times \mathbb{R}, \\
u & =0 \quad \text { on } \quad \partial B_{R} \times \mathbb{R},
\end{aligned}
$$

normalized by $\left\|\tilde{\varphi}_{R}(\cdot, 0)\right\|_{L^{\infty}\left(B_{R}\right)}=1$ (see $[16]$ ).

Theorem 9.5. Assume that (A), (B) and (H2)' hold. Then there is $R_{0}>0$ with the following property. Given any $\epsilon_{0}<\tilde{\epsilon}_{0}$, there exists positive constants $\delta$ and $\tilde{C}_{0}$ depending only on $\tilde{\epsilon}_{0}-\epsilon_{0}$ and the given quantities such that if

$$
\left\|a_{i j}-\tilde{a}_{i j}\right\|,\left\|a_{i}-\tilde{a}_{i}\right\|,\left\|b_{i}-\tilde{b}_{i}\right\|,\|a-\tilde{a}\|<\delta \quad(i, j=1, \ldots, N),
$$

where $\|\cdot\|$ means $\|\cdot\|_{L^{\infty}\left(\mathbb{R}^{N} \times \mathbb{R}\right)}$, then $\tilde{\varphi}_{R_{0}}$ satisfies the estimate

$$
\frac{\left\|\tilde{\varphi}_{R_{0}}(\cdot, t)\right\|_{L^{\infty}\left(B_{R_{0}}\right)}}{\left\|\tilde{\varphi}_{R_{0}}(\cdot, s)\right\|_{L^{\infty}\left(B_{R_{0}}\right)}} \geq \tilde{C}_{0} e^{\epsilon_{0}(t-s)} \quad\left(t \geq s \geq s_{0}\right) .
$$

This conclusion implies that (H1)' holds for the perturbed equation. If the perturbed coefficients satisfy a hypothesis similar to (B) and $\delta$ is chosen sufficiently small, then one can further prove the existence of a positive entire solution of (9.6) and the corresponding exponential separation as in Theorem 2.6 and Remark 2.7(i). 
To prove the above results, only minor modifications in the proofs of the corresponding theorems for (1.1) are needed. Indeed, our arguments in Sections 3-7 mostly rely on general tools, such as the maximum and comparison principles, Harnack inequality (including its extension in Theorem 3.2), and the week Green's functions. All these apply equally well in the more general setting thanks to the regularity assumptions we have made on the coefficients of (9.1). The only difference at this general level is that the Gaussian estimate on the week Green's function now reads as follows:

$0<\Gamma(x, t ; y, s) \leq e^{d_{1}(t-s)}(4 \pi(t-s))^{-N / 2} e^{-|x-y|^{2} / d_{2}(t-s)} \quad\left((x, y) \in \mathbb{R}^{N} \times \mathbb{R}^{N}\right)$, where $d_{1}$ and $d_{2}$ are positive constants depending on the given quantities (see [3]). This estimate is to replace (3.5) in arguments involving the Green's function, in particular in the proofs of Lemmas 3.3, 5.1, and 5.5.

The specific form of equation (1.1) was used only in the proof of Lemma 4.4 (the construction of a supersolution) and in the proof of Lemma 6.4 (the construction of a subsolution). In the latter, it is sufficient to replace $\Delta$ by $L(x, t)$ and the arguments go through in the more general setting. In the former, one needs to change the definition (4.15) of the function $\zeta_{R}$ as follows:

$$
\zeta_{R}(x, s)=q e^{-\epsilon_{0}\left(s-t_{0}\right)}+q e^{-\nu\left(|x|-\rho_{0}\right)} \quad\left(x \in \mathbb{R}^{N}, s \in\left[t_{0}, t\right]\right) .
$$

Here $q$ has the same meaning as in (4.15) and $\nu>0$ has to be chosen sufficiently small (but depending only on the given quantities) for $\zeta_{R}$ to be a subsolution. See [31, Proof of Lemma 2.4] for a computation which shows that such a choice of $\nu$ is possible (the nondivergence form of the equation is needed for this computation).

After the above modifications and other obvious changes (like replacements of references to the theorems in Section 2.2 by references to their counterparts in this section), the proofs of Theorems 2.1-2.4, and Proposition 2.5 become proofs of Theorems 9.1-9.3, and Proposition 9.4. The proof of Theorem 9.5 is a straightforward modification of the proof of Theorem 9.5 up to (7.2). The perturbation result of [16] used in the proof also applies in the more general setting of Theorem 9.5.

\section{References}

[1] S. Agmon, Lectures on exponential decay of solutions of second-order elliptic equations: bounds on eigenfunctions of $N$-body Schrödinger op- 
erators. Mathematical Notes, 29, Princeton University Press, Princeton, NJ; University of Tokyo Press, Tokyo, 1982.

[2] S. Angenent, Constructions with analytic semigroups and abstract exponential decay results for eigenfunctions. Progr. Nonlinear Differential Equations Appl., 35, Birkhuser, Basel, 1999.

[3] D. G. Aronson, Non-negative solutions of linear parabolic equations. Ann. Scuola Norm. Sup. Pisa, 22 (1968), 607-694.

[4] S.-N. Chow, K. Lu and J. Mallet-Paret, Floquet theory for parabolic differential equations. J. Differential Equations, 109 (1994),147-200.

[5] S.-N. Chow, K. Lu and J. Mallet-Paret, Floquet bundles for scalar parabolic equations. Arch. Rational Mech. Anal., 129 (1995), 245-304.

[6] D. Daners and P. K. Medina, Exponential stability, change of stability and eigenvalue problems for linear time-periodic equations on $\mathbb{R}^{N}$. Differential Integral Equations, 7 (1994),1265-1284.

[7] D. Daners, Principal eigenvalues for some periodic-parabolic operators on $\mathbb{R}^{N}$ and related topics. J. Differential Equations, 121 (1995),293-313.

[8] R. Hempel, A. Hinz and H. Kalf, On the essential spectrum of Schrödinger operators with spherically symmetric potentials. With a comment by J. Weidmann. Math. Ann., 277 (1987),197-211.

[9] P. Hess and P. Poláčik, Boundedness of prime periods of stable cycles and convergence to fixed points in discrete monotone dynamical systems. SIAM J. Math. Anal., 24 (1993),1312-1330.

[10] P. D. Hislop and I. M. Sigal, Introduction to Spectral Theory With applications to Schrödinger operators, volume 113 of Applied Mathematical Sciences. Springer, New York, 1996.

[11] J. Húska, Harnack inequality and exponential separation for oblique derivative problems on Lipschitz domains. J. Differential Equations, 226 (2006), 541-557.

[12] J. Húska, Exponential separation and principal Floquet bundles for linear parabolic equations on general bounded domains: divergence case. Indiana Univ. Math. J., 55 (2006),1015-1044. 
[13] J. Húska, Exponential separation and principal Floquet bundles for linear parabolic equations on general bounded domains: nondivergence case. Trans. Amer. Math. Soc., to appear.

[14] J. Húska and P. Poláčik, The principal Floquet bundle and exponential separation for linear parabolic equations. J. Dynam. Differential Equations, 24 (2004),1312-1330.

[15] J. Húska, P. Poláčik and M. V. Safonov, Principal eigenvalues, spectral gaps and exponential separation between positive and sign-changing solutions of parabolic equations. Proceedings of the 5th International Conference on Dynamical Systems and Differential Equations, Pomona 2004, Disc. Cont. Dynamical Systems, Supplement 2005, 427-435.

[16] J. Húska, P. Poláčik and M. Safonov, Harnack inequalities, exponential separation, and perturbations of principal Floquet bundles for linear parabolic equations. Ann. Inst. H. Poincaré Anal. Non Linéaire, 24 (2007),711-739.

[17] V. Hutson, W. Shen and G. T. Vickers, Estimates for the principal spectrum point for certain time-dependent parabolic operators. Proc. Amer. Math. Soc., (electronic), 129 (2001),1669-1679.

[18] N. V. Krylov, Lectures on Elliptic and Parabolic Equations in Hölder Spaces. American Mathematical Society, Graduate studies in mathematics, Providence, RI, 1996.

[19] O. A. Ladyzhenskaya, V. A. Solonnikov and N. N. Uralceva, Linear and Quasilinear Equations of Parabolic Type. American Mathematical Society, Translation of mathematical monographs, Providence, RI, 1968.

[20] G. M. Lieberman, Second order parabolic differential equations. World Scientific Publishing Co. Inc., River Edge, NJ, 1996.

[21] J. Mierczyński, Flows on order bundles. unpublished.

[22] J. Mierczyński, $p$-arcs in strongly monotone discrete-time dynamical systems. Differential Integral Equations, 7 (1994),1473-1494.

[23] J. Mierczyński, Globally positive solutions of linear PDEs of second order with Robin boundary conditions. J. Math. Anal. Appl., 209 (1997),47-59. 
[24] J. Mierczyński, Globally positive solutions of linear parabolic partial differential equations of second order with Dirichlet boundary conditions. J. Math. Anal. Appl., 226 (1998),326-347.

[25] J. Mierczyński, The principal spectrum for linear nonautonomous parabolic pdes of second order: Basic properties. J. Differential Equations, 168 (2000),453-476.

[26] J. Mierczyński and W. Shen, Exponential separation and principal Lyapunov exponent/spectrum for random/nonautonomous parabolic equations. J. Differential Equations, 191 (2003),175-205.

[27] J. Moser, A Harnack inequality for parabolic differential equations, Comm. Pure Appl. Math., 17 (1964),101-134; Correction in Comm. Pure Appl. Math., 20 (1967),231-236.

[28] M. Nishio, The uniqueness of positive solutions of parabolic equations of divergence form on an unbounded domain. Nagoya Math. J., 130 (1993),111-121.

[29] P. Poláčik, On uniqueness of positive entire solutions and other properties of linear parabolic equations. Discrete Contin. Dynamical Systems, 12 (2005),13-26.

[30] P. Poláčik, Estimates of solutions and asymptotic symmetry for parabolic equations on bounded domains. Arch. Rational Mech. Anal., 183 (2007),59-91.

[31] P. Poláčik, Symmetry properties of positive solutions of parabolic equations on $R^{N}$ : II. Entire solutions. Comm. Partial Differential Equations, 31 (2006), 1615-1638.

[32] P. Poláčik and I. Tereščák, Convergence to cycles as a typical asymptotic behavior in smooth strongly monotone discrete-time dynamical systems. Arch. Rational Mech. Anal., 116 (1992),339-360.

[33] P. Poláčik and I. Tereščák, Exponential separation and invariant bundles for maps in ordered Banach spaces with applications to parabolic equations. J. Dynamics Differential Equations, 5 (1993),279-303. Erratum: 6 (1994),245-246. 
[34] P. J. Rabier, Asymptotic behavior of the solutions of linear and quasilinear elliptic equations on $\mathbb{R}^{N}$. Trans. Amer. Math. Soc., 356 (2004),18891907.

[35] M. Reed and B. Simon, Methods of modern mathematical physics IV. Academic Press, New York, 1978.

[36] B. Simon, Schrödinger semigroups, Bull. Amer. Math. Soc. (N.S.), 7 (1982),447-526.

[37] W. Shen and Y. Yi, Almost automorphic and almost periodic dynamics in skew-product semiflows, Mem. Amer. Math. Soc., 136 (1998).

[38] I. Tereščák, Dynamical systems with discrete Lyapunov functionals. PhD thesis, Comenius University, 1994. 